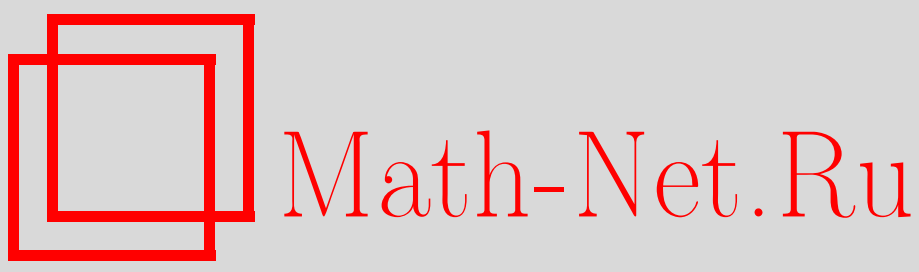

Я. Б. Воробец, О равномерном распределении орбит конечно порожденных групп и полугрупп изометрий плоскости, Матем. сб., 2004, том 195, номер 2, 17-40

DOI: https://doi.org/10.4213/sm799

Использование Общероссийского математического портала Math-Net.Ru подразумевает, что вы прочитали и согласны с пользовательским соглашением http://www . mathnet.ru/rus/agreement

Параметры загрузки:

IP: 34.229 .108 .108

26 апреля 2023 г., 13:31:54 
УДК 517.987.5

\author{
Я.Б. Воробец
}

\title{
О равномерном распределении орбит конечно порож,денных групп и полугрупп изометрий плоскости
}

\begin{abstract}
В статье рассматриваются действия на евклидовой плоскости изометриями свободных групп и свободных полугрупп с произвольньм числом образующих, а также свободных произведений групп порядка 2. Показано, что в типичном случае все орбиты действия равномерно распределены на плоскости. Действия, для которых нет равномерного распределения орбит, описаны явно.

Библиографиия: 5 названий.
\end{abstract}

\section{§1. Введение}

Пусть $P$ - аффинная евклидова плоскость. Обозначим через $\mathscr{G}$ группу изометрий плоскости $P$, т.е. преобразований, сохраняющих расстояния между точками. Каждый элемент групшы $\mathscr{G}$ является аффинным преобразованием. Через $\mathscr{G}_{+}$обозначим группу изометрий плоскости $P$, сохраняющих ориентацию, а через $\mathscr{G}_{0}-$ группу параллельных переносов. Повороты (вращения) плоскости образуют множество $\mathscr{G}_{+} \backslash \mathscr{G}_{0}$, а осевые и скользящие симметрии - множество $\mathscr{G} \backslash \mathscr{G}_{+}$. Группа $\mathscr{G}_{+}$ является подгруппой группы $\mathscr{G}$ индекса 2 . Группа $\mathscr{G}_{0}$ является нормальной подгруппой групп $\mathscr{G}_{\text {и }} \mathscr{G}_{+}$.

Пусть $G$ - счетная группа или полугруппа. Действием (полу)группы $G$ на плоскости $P$ посредством изометрий называется (полу)групповой гомоморфизм $d: G \rightarrow \mathscr{G}$. Opбитой $O_{d}(x)$ точки $x \in P$ относительно действия $d$ называется последовательность $\{d(g) x\}_{g \in G}$ точек плоскости, индексированная элементами (полу)группы $G$. Орбитой действия $d$ назьвается орбита какой-либо точки $x \in P$ относительно $d$.

В настоящей статье предполагается, что группа или полугруппа $G$ является конечно порожденной и, более того, снабжена фиксированным набором образующих $a_{1}, \ldots, a_{k}$. Это позволяет ввести на $G$ функцию длины. Длиной $|g|$ произвольного элемента $g \in G$ называется минимальное $m$ такое, что $g$ разлагается в произведение $g_{1} g_{2} \cdots g_{m}$, где сомножители берутся из последовательности $a_{1}, \ldots, a_{k}$, если $G$ - полугрупша, и из последовательности $a_{1}, a_{1}^{-1}, \ldots, a_{k}, a_{k}^{-1}$, если $G$-группа. Длина единичного элемента принимается равной нулю. Функция длины задает на (полу)группе $G$ частичный порядок, который переносится на каждую орбиту действия $d$ этой (полу)групшы. Мы используем этот порядок для определения понятия равномерно распределенной орбиты. Пусть $x$ - точка, а $E$ - подмножество плоскости $P$. Для любого натурального $n$ обозначим через $N_{d, x}^{(n)}(E)$ отношение числа элементов $g \in G$ длины $n$, для которых $d(g) x \in E$, к числу всех элементов 
длины $n$ в $G$. Орбита $O_{d}(x)$ называется равномерно распределенной на плоскости, если для любой пары $E_{1}, E_{2}$ измеримых по Жордану подмножеств плоскости, мера которых положительна,

$$
\lim _{n \rightarrow \infty} \frac{N_{d, x}^{(n)}\left(E_{1}\right)}{N_{d, x}^{(n)}\left(E_{2}\right)}=\frac{\mu\left(E_{1}\right)}{\mu\left(E_{2}\right)}
$$

где $\mu$ - мера Лебега на плоскости $P$.

Поскольку элементы $a_{1}, \ldots, a_{k}$ порождают (полу)группу $G$, действие $d$ однозначно определено изометриями $A_{1}=d\left(a_{1}\right), \ldots, A_{k}=d\left(a_{k}\right)$ и мы обозначаем его $G\left[A_{1}, \ldots, A_{k}\right]$. В качестве $G$ далее выступают свободная полугруппа $\mathrm{FSG}_{k}$ и свободная группа $\mathrm{FG}_{k}$ с $k$ образующими, а также свободное произведение $k$ групп порядка $2 \mathbb{Z}_{2}^{* k}=\left\langle a_{1}, \ldots, a_{k} \mid a_{1}^{2}=\cdots=a_{k}^{2}=1\right\rangle$. Заметим, что действия $\mathrm{FG}_{k}\left[A_{1}, \ldots, A_{k}\right]$ и $\mathrm{FSG}_{k}\left[A_{1}, \ldots, A_{k}\right]$ сушествуют для любых $A_{1}, \ldots, A_{k} \in \mathscr{G}$. Действие $\mathbb{Z}_{2}^{* k}\left[A_{1}, \ldots, A_{k}\right]$ сушествует, если $A_{1}, \ldots, A_{k}$ - инволюции. В настоящей статье исследуются асимптотические свойства средних $N_{d, x}^{(n)}(E)$, в частности вопрос о равномерном распределении орбит, для перечисленных выше действий $d$. Соответствующая задача была поставлена (в ряду других задач) в статье В.И. Арнольда и А. Л. Крылова [1]. Исследования были начаты Д. Кажданом [2] и И. Гиваршем [3], а затем продолжены автором в работах [4], [5]; полученные результаты подытоживаются сформулированньми ниже теоремами 1.1 и 1.2.

Для формулировки теорем понадобится еще одно определение. Пусть $Q$ обозначает полосу (область, ограниченную двумя параллельными прямыми), квадрат или правильньй треугольник на плоскости $P$. Отразим фигуру $Q$ относительно каждой из ограничивающих ее прямых, потом проделаем то же с отражениями фигуры $Q$ и так далее до бесконечности. В итоге получим разбиение плоскости $P$ посредством счетного числа прямых на фигуры, конгруэнтные $Q$. Мы будем называть это разбиение решеткой, порожденной фигурой $Q$, и обозначать $\mathscr{L}_{Q}$. Скажем, что изометрия $A \in \mathscr{G}$ переводит решетку $\mathscr{L}_{Q}$ в себя, если она переводит друг в друга прямые, определяюшие разбиение $\mathscr{L}_{Q}$.

Tеорема $1.1[5]$. Пусть $A_{1}, \ldots, A_{k} \in \mathscr{G}$. Положим

$$
d=\mathrm{FSG}_{2 k}\left[A_{1}, A_{1}^{-1}, \ldots, A_{k}, A_{k}^{-1}\right]
$$

или $d=\mathrm{FG}_{k}\left[A_{1}, \ldots, A_{k}\right]$, или (при условии, что $A_{1}, \ldots, A_{k}-$ инволючии) $d=\mathbb{Z}_{2}^{* k}\left[A_{1}, \ldots, A_{k}\right]$. Если изометрии $A_{1}, \ldots, A_{k}$ не имеют общей неподвижной точки и не переводят в себя одну и ту же решетку вида $\mathscr{L}_{Q}$, әде $Q$ - полоса, квадрат или правильный треугольник, то все орбить действия $d$ равномерно распределены на плоскости. Более того, для любого измеримого по Жордану множества Е положительной меры и любой точки $x$ при достаточно больших значениях $n$ имеют место оченки

$$
I_{1} \mu(E) n^{-1} \leqslant N_{d, x}^{(n)}(E) \leqslant I_{2} \mu(E) n^{-1}
$$

әде $I_{1}, I_{2}$ - положительные постоянные, зависящие лишь от действия $d$. 
Теорема 1.2 [4]. Пусть $A_{1}, A_{2} \in \mathscr{G}_{+}$. Если изометрии $A_{1}, A_{2}$ не имеют общей неподвижсной точки и не переводят в себя одну и ту же решетку вида $\mathscr{L}_{Q}$, где $Q$ - полоса, квадрат или правильный треугольник, то все орбиты действия $d=\mathrm{FSG}_{2}\left[A_{1}, A_{2}\right]$ равномерно распределеньи на плоскости.

Основным результатом настоящей работы являются следующие две теоремы, первая из которых усиливает теорему 1.1, а вторая обобщает теорему 1.2 .

Теорема 1.3. Пусть $A_{1}, \ldots, A_{k} \in \mathscr{G}$. Полољимм

$$
d=\mathrm{FSG}_{2 k}\left[A_{1}, A_{1}^{-1}, \ldots, A_{k}, A_{k}^{-1}\right]
$$

или $d=\mathrm{FG}_{k}\left[A_{1}, \ldots, A_{k}\right]$, или (при условии, что $A_{1}, \ldots, A_{k}-$ инволючии) $d=\mathbb{Z}_{2}^{* k}\left[A_{1}, \ldots, A_{k}\right]$. Если изометрии $A_{1}, \ldots, A_{k}$ не имеют общей неподвижной точки и не переводят в себя одну и ту же решетку вида $\mathscr{L}_{Q}$, где $Q$ полоса, квадрат или правильный треугольник, то найдется $I_{0}>0$ такое, что $\lim _{n \rightarrow \infty} n N_{d, x}^{(n)}(E)=I_{0} \mu(E)$ для любого измеримого по Жордану множсества $E \subset P$ и любой точки $x \in P$. При этом все орбить действия $d$ равномерно распределенъ на плоскости.

Теорема 1.4. Пусть $A_{1}, \ldots, A_{k} \in \mathscr{G}$. Если изометрии $A_{1}, \ldots, A_{k}$ не имеют общей неподвижной точки и не переводят в себя одну и ту же решетку вида $\mathscr{L}_{Q}$, где $Q$ - полоса, квадрат или правильный треугольник, то действие $d=\mathrm{FSG}_{k}\left[A_{1}, \ldots, A_{k}\right]$ удовлетворяет одному из следующих двух условий:

1) найдется $I_{0}>0$ такое, что $\lim _{n \rightarrow \infty} n N_{d, x}^{(n)}(E)=I_{0} \mu(E)$ для любого измеримого по Жордану множсества $E \subset P$ и любой точки $x \in P$; при этом все орбиты действия $d$ равномерно распределены на плоскости;

2) найдется $I_{0}>0$ такое, что для любого ограниченного множества $E \subset P$ и любой точки $x \in P$ последовательность $\left\{\exp \left(I_{0} n\right) N_{d, x}^{(n)}(E)\right\}$ ограничена.

Условие 2) выполнено лишь в следующих случаях:

(i) $A_{1}, \ldots, A_{k}$ - параллельные переносы на векторы, сумма которых не равна нулю;

(ii) каждая из изометрий $A_{1}, \ldots, A_{k}$ является либо параллельнымм переносом, либо осевой или скользящей симметрией, ось которой параллельна фиксированной прямой $l$, причем для некоторой ( и тогда для любой) точки $x \in P$ сумма векторов $A_{j} x-x, j=1, \ldots, k$, не ортогональна прямой $l$.

Доказательству теорем 1.3 и 1.4 посвящены $\S \S 2,3$ настоящей статьи, а пока что прокомментируем эти утверждения. Если изометрии $A_{1}, \ldots, A_{k}$ имеют общуюнеподвижную точку, то каждая орбита действия $d$ лежит на окружности с центром в этой точке. Если изометрии $A_{1}, \ldots, A_{k}$ переводят в себя решетку, порожденную полосой, то каждая орбита содержится в объединении счетного числа параллельных прямых, расстояния между которыми не менее некоторого $\varepsilon>0$. Если же эти изометрии переводят в себя решетку, порожденную квадратом или правильным треугольником, то точки каждой орбиты образуют дискретное подмножество плоскости. Во всех перечисленных случаях орбиты действия $d$ нигде не плотны на 
плоскости. С другой стороны, если орбиты действия $d$ равномерно распределены на плоскости $P$, то они всюду плотны на $P$. Согласно общей гипотезе, высказанной в работе [1], произвольная орбита действия $d$ равномерно распределена на плоскости, как только она всюду плотна там. Как видим, теорема 1.3 (как и теорема 1.1) подтверждает эту гипотезу для действий групп $\mathrm{FG}_{k}$ и $\mathbb{Z}_{2}^{* k}$, а теорема 1.4 подтверждает ее для действий полугруппы $\mathrm{FSG}_{k}$ во всех случаях, кроме случаев (i) и (ii).

Автор благодарен Р.И. Григорчуку за обсуждение результатов работы и постоянную поддержку.

\section{§2. Равномерное распределение}

Для каждой изометрии $A \in \mathscr{G}$ обозначим через $u[A]$ линейный оператор, действуюший на функции на плоскости $P$ по формуле $(u[A] f)(x)=f(A x), x \in P$. Ограничение $u[A]$ на пространство $L_{2}(P, \mu)$ является унитарным оператором. Через $\mathscr{U}$ обозначим класс операторов, образованный конечньми линейными комбинациями операторов вида $u[A]$. Поскольку $u[A] u[B]=u[B A]$ для любых $A, B \in \mathscr{G}$, класс $\mathscr{U}$ является алгеброй операторов.

Обозначим через $F$ преобразование Фурье в $\mathbb{R}^{2}$. Мы рассматриваем $F$ как унитарный автоморфизм пространства $L_{2}\left(\mathbb{R}^{2}\right)$. Действие операторов $F$ и $F^{-1}$ на функцию $f \in L_{1}\left(\mathbb{R}^{2}\right) \cap L_{2}\left(\mathbb{R}^{2}\right)$ задается формулами

$$
\begin{gathered}
(F f)(\lambda)=\frac{1}{2 \pi} \int_{\mathbb{R}^{2}} e^{-i(\lambda, x)} f(x) d x, \\
\left(F^{-1} f\right)(x)=(F f)(-x) .
\end{gathered}
$$

Пусть $\mathscr{F}$ обозначает класс функций $f \in L_{1}\left(\mathbb{R}^{2}\right) \cap L_{2}\left(\mathbb{R}^{2}\right)$, для которых Фурьеобраз $F f$ дифференцируем и финитен. Введем на плоскости $P$ декартовы координаты $\xi$. Мы рассматриваем $\xi$ как изометричное отображение $P$ на евклидово пространство $\mathbb{R}^{2}$. Координаты $\xi$ позволяют рассматривать произвольную функцию $f$ на плоскости $P$ как функцию на $\mathbb{R}^{2}$ (отождествив ее с функцией $f \xi^{-1}$ ). В частности, можно рассматривать $\mathscr{F}$ как класс функций на плоскости $P$.

Лемма 2.1 [5]. Класс $\mathscr{F}$ не зависит от выбора декартовых координат на плоскости $P$ и инвариантен относительно операторов из алгебры $\mathscr{U}$. Для любого измеримого по Жордану множества $E \subset P$ и любого $\varepsilon>0$ найдется пара функиий $f_{+}, f_{-} \in \mathscr{F}$ таких, что $f_{-} \leqslant \chi_{E} \leqslant f_{+}$на всей плоскости и $\int\left(f_{+}-f_{-}\right) d \mu<\varepsilon$.

Символом окружности $S^{1}$ мы обозначаем факторпространство $\mathbb{R} / 2 \pi \mathbb{Z}$, снабженное естественной топологией и мерой Лебега. Важную роль в дальнейшем играет отображение $\Phi: S^{1} \rightarrow \mathbb{R}^{2}$, заданное формулой $\Phi(t)=(\cos t, \sin t), t \in \mathbb{R} / 2 \pi \mathbb{Z}$. Для любого $R>0$ отображение $R \Phi$ осуществляет гомеоморфизм $S^{1}$ на окружность радиуса $R$ в $\mathbb{R}^{2}$ с центром в начале координат. Пусть $f \in L_{1}\left(\mathbb{R}^{2}\right) \cap L_{2}\left(\mathbb{R}^{2}\right)$. Фурье-образ $F f$ функции $f$ непрерывен. Для каждого $R>0$ обозначим через $f_{R}$ непрерывную функцию на $S^{1}$, задаваемую формулой $f_{R}(t)=(F f)(R \Phi(t)), t \in S^{1}$. 
Функцию $f_{R}$ назовем радиальной компонентой функции $f$, соответствующей радиусу $R$.

Декартовы координаты $\xi$ позволяют рассматривать изометрии из группы $\mathscr{G}$ как преобразования $\mathbb{R}^{2}$, а операторы из алгебры $\mathscr{U}$, соответственно, как операторы, действующие на функции на $\mathbb{R}^{2}$. Радиальным оператором $u_{R}$, соответствующим оператору $u \in \mathscr{U}$ и радиусу $R>0$, назовем ограниченный линейный оператор в $L_{2}\left(S^{1}\right)$, который для любой функции $f \in L_{1}\left(\mathbb{R}^{2}\right) \cap L_{2}\left(\mathbb{R}^{2}\right)$ переводит ее радиальную компоненту $f_{R}$ в радиальную компоненту $(u f)_{R}$ функции $u f$. Отметим, что определение оператора $u_{R}$ зависит от системы координат $\xi$.

Лемма 2.2 [5]. Для любого оператора $u \in \mathscr{U}$ и числа $R>0$ радиальный оператор $u_{R}$ существует и единственен. Для каждого $R>0$ соответствие $u \mapsto u_{R}$ является гомоморфизмом алгебры $\mathscr{U}$ в алгебру линейных операторов в $L_{2}\left(S^{1}\right)$. Для любого $A \in \mathscr{G}$ и любого $R>0$ радиальный оператор $u[A]_{R}$ унитарен. Если изометрия $A$ оставляет неподвижным начало координат, то $u[A]_{R} h=h \Phi^{-1} A \Phi$ для любого $R>0 u h \in L_{2}\left(S^{1}\right)$. Eсли $A-$ параллельный перенос на вектор $v \in \mathbb{R}^{2}$, то $u[A]_{R}$ есть оператор умножения на функцию $e^{i R(\Phi, v)}$.

Пусть $G$ - конечно порожденная группа или полугруппа с выделенным набором образуюших, а $d$ - действие $G$ на плоскости $P$ посредством изометрий. Сопоставим действию $d$ последовательность усредняющих операторов $C^{(0)}, C^{(1)}, C^{(2)}, \ldots$, принадлежаших алгебре $\mathscr{U}$. Оператор $C^{(n)}$ задается равенством

$$
C^{(n)}=m_{n}^{-1} \sum_{|g|=n} u[d(g)]
$$

где $m_{n}$ - число элементов длины $n$ в $G$. Если $G$ - полугруппа без единичного элемента, то оператор $C^{(0)}$ не определен.

ПрЕДЛОЖЕНИЕ 2.3. Пусть $C_{R}^{(n)}, R>0, n=0,1, \ldots,-$ pадиальные оператоpьl, соответствующие усредняющим операторам $C^{(0)}, C^{(1)}, \ldots$ в некоторой системе координат $\xi$. Предположим, что операторь $C_{R}^{(n)}$ обладают следующими свойствами:

(P1) найдутся положительнье постоянные $I_{1}, I_{2}$ и $R_{0}$ такие, что

$$
\left\|C_{R}^{(n)}\right\| \leqslant I_{1}\left(1-I_{2} R^{2}\right)^{n}, \quad 0<R \leqslant R_{0} ;
$$

(P1b) найдутся положительнье постояннье $I_{0}$ и $R_{0}$ такие, что

$$
n \int_{0}^{R_{0}} R\left(C_{R}^{(n)} 1,1\right) d R \rightarrow I_{0}, \quad n \rightarrow \infty
$$

(P2) для любьх $R_{1}$ u $R_{2}, 0<R_{1}<R_{2}$, найдутся постоянные $I>0 u$ $\rho \in(0,1)$ mакие, что $\left\|C_{R}^{(n)}\right\| \leqslant I \rho^{n}$ nрu $R_{1} \leqslant R \leqslant R_{2}$.

Тогда

$$
\lim _{n \rightarrow \infty} n N_{d, x}^{(n)}(E)=(2 \pi)^{-2} I_{0} \mu(E)
$$

для любого измеримого по Жордану множества $E \subset P$ и любой точки $x \in P$. Как следствие, каждая орбита действия d равномерно распределена на плоскости. 
ДокАЗАТЕльство. Поскольку $\left|\left(C_{R}^{(n)} 1,1\right)\right| \leqslant 2 \pi\left\|C_{R}^{(n)}\right\|$, из свойства (Р2) следует, что для любого $R_{1}>0$ величина $n \int_{R_{0}}^{R_{1}} R\left(C_{R}^{(n)} 1,1\right) d R$ стремится к нулю при $n \rightarrow \infty$. Тем самым, свойство (P1b) не нарушится, если постоянную $R_{0}$ выбирать произвольным образом, причем значение постоянной $I_{0}$ от этого произвола не зависит. Это позволяет считать, что постоянная $R_{0}$ в условиях (P1) и (P1b) одна и та же, причем $I_{2} R_{0}^{2}<1$. Тогда при $0<R \leqslant R_{0}$ имеют место оценки $0<1-I_{2} R^{2} \leqslant \exp \left(-I_{2} R^{2}\right) ;$ как следствие, $\left\|C_{R}^{(n)}\right\| \leqslant I_{1} \exp \left(-I_{2} n R^{2}\right)$.

Покажем, что наличие свойств (P1), (P1b), (P2) не зависит от системы координат, в которой вычисляются радиальные операторы $C_{R}^{(n)}$, как не зависит от нее и значение постоянной $I_{0}$ в условии $(\mathrm{P} 1 \mathrm{~b})$. Пусть $\xi_{1}-$ декартовы координаты на плоскости $P$, отличные от $\xi$. Пусть $\widetilde{C}_{R}^{(n)}, R>0, n=0,1, \ldots,-$ семейство радиальных операторов, соответствующих операторам $C^{(0)}, C^{(1)}, \ldots$ в системе координат $\xi_{1}$. Положим $A=\xi^{-1} \xi_{1}$. Очевидно, $A \in \mathscr{G}$. Легко проверить, что оператор $C^{(n)}$ действует на функции на $\mathbb{R}^{2}$ относительно координат $\xi_{1}$ точно так же, как оператор $u[A]^{-1} C^{(n)} u[A]$ действует на них относительно координат $\xi$. Отсюда следует, что $\widetilde{C}_{R}^{(n)}=u[A]_{R}^{-1} C_{R}^{(n)} u[A]_{R}$, где оператор $u[A]_{R}$ вычисляется в системе координат $\xi$. Поскольку оператор $u[A]_{R}$ унитарен, имеем $\left\|\widetilde{C}_{R}^{(n)}\right\|=\left\|C_{R}^{(n)}\right\|$ и $\left(\widetilde{C}_{R}^{(n)} 1,1\right)=\left(C_{R}^{(n)}\left(u[A]_{R} 1\right), u[A]_{R} 1\right)$. Ввиду первого равенства наличие свойств (P1) и (Р2) не зависит от выбора системы координат. Из второго равенства следует, что

$$
\left|\left(\widetilde{C}_{R}^{(n)} 1,1\right)-\left(C_{R}^{(n)} 1,1\right)\right| \leqslant 4 \pi\left\|C_{R}^{(n)}\right\| \sup \left|u[A]_{R} 1-1\right| .
$$

Согласно лемме $2.2 u[A]_{R} 1=e^{i R(\Phi, v)}$, где вектор $v \in \mathbb{R}^{2}$ зависит лишш от $A$ и $\xi$. Тогда при $0<R \leqslant R_{0}$ имеем $\sup \left|u[A]_{R} 1-1\right| \leqslant I_{*} R$, где постоянная $I_{*}>0$ зависит лишш от $A, \xi$ и $R_{0}$. При этом $\left|\left(\widetilde{C}_{R}^{(n)} 1,1\right)-\left(C_{R}^{(n)} 1,1\right)\right| \leqslant 4 \pi I_{*} R\left\|C_{R}^{(n)}\right\| \leqslant$ $4 \pi I_{*} I_{1} R \exp \left(-I_{2} n R^{2}\right)$. Как следствие,

$$
\begin{aligned}
& n \int_{0}^{R_{0}} R\left|\left(\widetilde{C}_{R}^{(n)} 1,1\right)-\left(C_{R}^{(n)} 1,1\right)\right| d R \leqslant 4 \pi I_{*} I_{1} n \int_{0}^{R_{0}} R^{2} \exp \left(-I_{2} n R^{2}\right) d R \\
& \quad=4 \pi I_{*} I_{1} n^{-1 / 2} \int_{0}^{n^{1 / 2} R_{0}} R^{2} \exp \left(-I_{2} R^{2}\right) d R \\
& \quad \leqslant 4 \pi I_{*} I_{1} n^{-1 / 2} \int_{0}^{\infty} R^{2} \exp \left(-I_{2} R^{2}\right) d R
\end{aligned}
$$

что стремится к нулю при $n \rightarrow \infty$. Тем самьм, операторы $\widetilde{C}_{R}^{(n)}$, как и операторы $C_{R}^{(n)}$, обладают свойством (P1b), причем с тем же значением постоянной $I_{0}$.

Выберем точку $x \in P$. Ввиду сказанного выше можно считать, не ограничивая общности, что $x$ - начало координат в системе $\xi$. Возьмем произвольную функцию $f \in \mathscr{F}$. Для любого натурального $n$ положим

$$
\begin{aligned}
\alpha_{n} & =(2 \pi)^{-2} n \int_{0}^{R_{0}} R\left(C_{R}^{(n)} 1,1\right) d R \\
\beta_{n}(f) & =n^{3 / 2}\left(\left(C^{(n)} f\right)(x)-n^{-1} \alpha_{n} \int f d \mu\right) .
\end{aligned}
$$


При этом

$$
\left(C^{(n)} f\right)(x)=n^{-1} \alpha_{n} \int f d \mu+n^{-3 / 2} \beta_{n}(f) .
$$

Согласно лемме $2.1 C^{(n)} f \in \mathscr{F}$. Рассматривая функцию $C^{(n)} f$ как функцию на $\mathbb{R}^{2}$ (относительно координат $\xi$ ), имеем:

$$
\left(C^{(n)} f\right)(x)=\frac{1}{2 \pi} \int_{\mathbb{R}^{2}}\left(F\left(C^{(n)} f\right)\right)(\lambda) d \lambda=\frac{1}{2 \pi} \int_{0}^{\infty} R\left(C_{R}^{(n)} f_{R}, 1\right) d R .
$$

Учитывая то, что $\int f d \mu=2 \pi(F f)(0)$ и $\left|\left(C_{R}^{(n)} h, 1\right)\right| \leqslant 2 \pi\left\|C_{R}^{(n)}\right\| \sup |h|$ для любой функции $h \in L_{2}\left(S^{1}\right)$, приходим к оценке $\left|\beta_{n}(f)\right| \leqslant J_{1, n}+J_{2, n}$, где

$$
\begin{aligned}
& J_{1, n}=n^{3 / 2} \int_{0}^{R_{0}} R\left\|C_{R}^{(n)}\right\| \sup \left|f_{R}-(F f)(0)\right| d R \\
& J_{2, n}=n^{3 / 2} \int_{R_{0}}^{\infty} R\left\|C_{R}^{(n)}\right\| \sup \left|f_{R}\right| d R .
\end{aligned}
$$

Поскольку $f \in \mathscr{F}$, найдутся постоянные $R_{1}>R_{0}$ и $I_{f}>0$ такие, что $f_{R}=0$ при $R \geqslant R_{1}$ и $\sup \left|f_{R}-(F f)(0)\right| \leqslant I_{f} R$ при $0<R \leqslant R_{0}$. Так как

$$
\sup \left|f_{R}\right| \leqslant \sup |F f| \leqslant \frac{1}{2 \pi} \int|f| d \mu
$$

для любого $R>0$, из свойства $(\mathrm{P} 2)$ следует, что $J_{2, n} \rightarrow 0$ при $n \rightarrow \infty$. Далее,

$$
J_{1, n} \leqslant n^{3 / 2} \int_{0}^{R_{0}} I_{f} I_{1} R^{2} \exp \left(-I_{2} n R^{2}\right) d R \leqslant I_{f} I_{1} \int_{0}^{\infty} R^{2} \exp \left(-I_{2} R^{2}\right) d R
$$

Тем самым, последовательность $\left\{\beta_{n}(f)\right\}$ ограничена. Согласно свойству (P1b) $\lim _{n \rightarrow \infty} \alpha_{n}=(2 \pi)^{-2} I_{0}>0$. Тогда

$$
n\left(C^{(n)} f\right)(x)=\alpha_{n} \int f d \mu+n^{-1 / 2} \beta_{n}(f) \rightarrow(2 \pi)^{-2} I_{0} \int f d \mu, \quad n \rightarrow \infty .
$$

Из леммы 2.1 следует, что для любого измеримого по Жордану множества $E \subset P$ и любого $\varepsilon>0$ найдутся функции $f_{+}, f_{-} \in \mathscr{F}$ такие, что $f_{-} \leqslant \chi_{E} \leqslant f_{+}$на всей плоскости,

$$
\int f_{+} d \mu<\mu(E)+\varepsilon, \quad \int f_{-} d \mu>\mu(E)-\varepsilon .
$$

Очевидно, $\left(C^{(n)} \chi_{E}\right)(x)=N_{d, x}^{(n)}(E)$ для любого натурального $n$, поэтому

$$
\left(C^{(n)} f_{-}\right)(x) \leqslant N_{d, x}^{(n)}(E) \leqslant\left(C^{(n)} f_{+}\right)(x)
$$


Согласно сказанному вьше

$$
\begin{aligned}
& \lim _{n \rightarrow \infty} n\left(C^{(n)} f_{+}\right)(x)=(2 \pi)^{-2} I_{0} \int f_{+} d \mu, \\
& \lim _{n \rightarrow \infty} n\left(C^{(n)} f_{-}\right)(x)=(2 \pi)^{-2} I_{0} \int f_{-} d \mu .
\end{aligned}
$$

Как следствие, все предельные точки последовательности $\left\{(2 \pi)^{2} I_{0}^{-1} n N_{d, x}^{(n)}(E)\right\}$ лежат на отрезке

$$
\left[\int f_{-} d \mu, \int f_{+} d \mu\right] \subset[\mu(E)-\varepsilon, \mu(E)+\varepsilon] .
$$

Поскольку $\varepsilon$ выбиралось произвольно, $\lim _{n \rightarrow \infty} n N_{d, x}^{(n)}(E)=(2 \pi)^{-2} I_{0} \mu(E)$. Ввиду произвольности выбора множества $E$ отсюда следует равномерная распределенность орбиты $O_{d}(x)$ на плоскости. Наконец, как было отмечено ранее, постоянная $I_{0}$ не зависит от выбора системы координат $\xi$, в частности от точки $x$, а зависит от действия $d$.

Далее в этом параграфе будут получены достаточные условия того, что абстрактное семейство $C_{R}^{(n)}, R>0, n=0,1, \ldots$, линейных ограниченных операторов в пространстве $L_{2}\left(S^{1}\right)$ обладает свойствами $(\mathrm{P} 1),(\mathrm{P} 1 \mathrm{~b})$ и $(\mathrm{P} 2)$. Мы рассмотрим случай, когда $C_{R}^{(n)}=p_{n}\left(C_{R}\right)$, где $\left\{C_{R}\right\}_{R>0}-$ некое семейство ограниченных операторов в пространстве $L_{2}\left(S^{1}\right)$, а $p_{0}, p_{1}, \ldots$ - многочлены с вещественными коэффициентами. Свойства операторов $C_{R}, R>0$, полезные для наших целей, таковы:

(V1) найдутся положительные постоянные $I_{1}$ и $R_{0}$ такие, что $\left\|C_{R}\right\| \leqslant 1-I_{1} R^{2}$ при $0<R \leqslant R_{0}$;

$\left(\mathrm{V} 1^{\prime}\right)$ найдутся положительные постоянные $I_{1}, R_{0}$ и натуральное число $m$ такие, что $\left\|C_{R}^{m}\right\| \leqslant 1-I_{1} R^{2}$ при $0<R \leqslant R_{0}$;

(V1b) найдутся постоянные $I_{0}>1, R_{0}>0$, измеримая функция $g$ на $S^{1}$ и отображение $\left(0, R_{0}\right] \ni R \mapsto h_{R} \in L_{2}\left(S^{1}\right)$ такие, что $I_{0}^{-1} \leqslant g \leqslant I_{0}$ на всей окружности, $\left\|h_{R}-1\right\| \leqslant I_{0} R$ и $\left\|C_{R} h_{R}-\left(1-g R^{2}\right) h_{R}\right\| \leqslant I_{0} R^{3}$ при $0<R \leqslant R_{0}$, а кроме того, для любого $R \in\left(0, R_{0}\right]$ оператор $C_{R}$ коммутирует с оператором умножения на функцию $g$;

(V2) $\left\|C_{R}\right\|<1$ для любого $R>0$;

$\left(\mathrm{V} 2^{\prime}\right)$ для любого $R_{1}>0$ найдется натуральное $M$ такое, что $\left\|C_{R}^{M}\right\|<1$ при $0<R \leqslant R_{1}$.

Пусть многочлены $p_{0}, p_{1}, p_{2}, \ldots$ удовлетворяют следующим условиям:

(W1) найдутся положительные постоянные $I_{2}, I_{3}$ и $\varepsilon$ такие, что

$$
\max \left(\left|p_{n}(1-z)\right|,\left|p_{n}(-1+z)\right|\right) \leqslant I_{2}\left(1-I_{3} z\right)^{n}, \quad 0 \leqslant z \leqslant \varepsilon ;
$$

(W1b) найдутся положительные постоянные $I_{4}, I_{5}, I_{6}$ и $\varepsilon$ такие, что

$$
\left|p_{n}(1-z)-I_{4}\left(1-I_{5} z\right)^{n}\right| \leqslant I_{6}\left(z+n z^{2}\right), \quad 0 \leqslant z \leqslant \varepsilon ;
$$

(W2) для любого $z_{0} \in(0,1)$ найдутся положительные постоянные $I$ и $\rho$ такие, что $\left|p_{n}(z)\right| \leqslant I \rho^{n},-z_{0} \leqslant z \leqslant z_{0}$. 
ПРЕДЛОЖЕНИЕ 2.4. Пусть $\left\{C_{R}\right\}_{R>0}$ - семейство ограниченных самосопряженных операторов в $L_{2}\left(S^{1}\right)$, непрерьвно зависящих от параметра $R$, a $p_{0}, p_{1}, \ldots$ - последовательность многочленов с вещественными коэффициентами. Если семейство $\left\{C_{R}\right\}_{R>0}$ обладает свойствами (V1), (V1b) и (V2), а многочлены $p_{0}, p_{1}, \ldots$ удовлетворяют условиям (W1), (W1b) и (W2), то семейство операторов $C_{R}^{(n)}=p_{n}\left(C_{R}\right), R>0, n=0,1, \ldots$, обладает свойствами (P1), (P1b) и (P2).

ДокАЗАТЕЛЬСТво. Наличие свойств (P1) и (Р2) устанавливается дословно так же, как и в доказательстве предложения 2.4 из [5]. Нужно отметить, что сослаться непосредственно на упомянутое предложение нельзя, так как в нем, в отличие от доказываемого предложения, вместо условий (V1b) и (W1b) налагаются другие условия, обозначаемые (V1a) и (W1a). Однако при проверке свойств (P1), (P2) используются лишш условия (V1), (V2) и (W1), (W2).

Проверим наличие свойства (P1b). Будем придерживаться тех же обозначений, что и в формулировках условий (V1), (V1b), (V2) и (W1), (W1b), (W2). Не ограничивая общности, можно считать, что постоянная $R_{0}$ в условиях (V1) и (V1b) одна и та же, постоянная $\varepsilon$ в условиях (W1) и (W1b) одна и та же и $R_{0}^{3 / 2} \leqslant \varepsilon<1$, $\max \left(I_{1} R_{0}^{1 / 2}, I_{5} R_{0}^{3 / 2}, I_{0} I_{5} R_{0}^{2}\right)<1$. Кроме того, ввиду свойства $(\mathrm{P} 1)$ можно считать, что при $0<R \leqslant R_{0}$ для любого целого $n \geqslant 0$ верна оценка $\left\|p_{n}\left(C_{R}\right)\right\| \leqslant$ $I_{7} \exp \left(-I_{8} n R^{2}\right)$, где $I_{7}, I_{8}$ - положительные постоянные.

Пусть $R \in\left(0, R_{0}\right]$. Поскольку оператор $C_{R}$ самосопряжен и $\left\|C_{R}\right\|<1$, его спектр содержится внутри отрезка $[-1,1]$. Из спектральной теоремы следует, что гильбертово пространство $L_{2}\left(S^{1}\right)$ разлагается в прямую сумму двух подпространств $H_{R}^{+}$и $H_{R}^{-}$, инвариантных относительно оператора $C_{R}$, причем спектры ограничений оператора $C_{R}$ на эти подпространства заключены внутри отрезков $\left[1-R^{3 / 2}, 1\right]$ и $\left[-1,1-R^{3 / 2}\right]$ соответственно. Более того, пространства $H_{R}^{+}$и $H_{R}^{-}$ инвариантны относительно любого ограниченного оператора, коммутирующего с $C_{R}$. В частности, эти пространства инвариантны относительно умножения на функцию $g$. Функция $h_{R}$ представляется в виде $h_{R}^{+}+h_{R}^{-}$, где $h_{R}^{+} \in H_{R}^{+}, h_{R}^{-} \in H_{R}^{-}$. При этом $C_{R} h_{R}^{+}-\left(1-g R^{2}\right) h_{R}^{+} \in H_{R}^{+}, C_{R} h_{R}^{-}-\left(1-g R^{2}\right) h_{R}^{-} \in H_{R}^{-}$, откуда следует, что

$$
\left\|C_{R} h_{R}^{+}-\left(1-g R^{2}\right) h_{R}^{+}\right\| \leqslant\left\|C_{R} h_{R}-\left(1-g R^{2}\right) h_{R}\right\| \leqslant I_{0} R^{3} .
$$

Далее, $\left(C_{R} h_{R}^{+}, h_{R}^{+}\right) \leqslant\left(h_{R}^{+}, h_{R}^{+}\right)$и $\left(C_{R} h_{R}^{-}, h_{R}^{-}\right) \leqslant\left(1-R^{3 / 2}\right)\left(h_{R}^{-}, h_{R}^{-}\right)$, поэтому

$$
\left(C_{R} h_{R}, h_{R}\right)=\left(C_{R} h_{R}^{+}, h_{R}^{+}\right)+\left(C_{R} h_{R}^{-}, h_{R}^{-}\right) \leqslant\left(h_{R}, h_{R}\right)-R^{3 / 2}\left(h_{R}^{-}, h_{R}^{-}\right) .
$$

В то же время, $\left|\left(C_{R} h_{R}, h_{R}\right)-\left(\left(1-g R^{2}\right) h_{R}, h_{R}\right)\right| \leqslant I_{0} R^{3}\left\|h_{R}\right\|$, поэтому

$$
\left(C_{R} h_{R}, h_{R}\right) \geqslant\left(1-I_{0} R^{2}\right)\left(h_{R}, h_{R}\right)-I_{0} R^{3}\left\|h_{R}\right\|
$$

Как следствие,

$$
\left\|h_{R}^{-}\right\|^{2} \leqslant I_{0} R^{1 / 2}\left\|h_{R}\right\|^{2}+I_{0} R^{3 / 2}\left\|h_{R}\right\| .
$$

Учитывая то, что $\left\|h_{R}\right\| \leqslant\|1\|+\left\|h_{R}-1\right\| \leqslant(2 \pi)^{1 / 2}+I_{0} R_{0}$, приходим к оценке вида $\left\|h_{R}^{-}\right\| \leqslant I_{9} R^{1 / 4}$, где $I_{9}$ - положительная постоянная, выражаюшаяся через 
$I_{0}$ и $R_{0}$. Наконец, $\left\|h_{R}^{+}-1\right\| \leqslant\left\|h_{R}-1\right\|+\left\|h_{R}^{-}\right\| \leqslant\left(I_{0} R_{0}^{3 / 4}+I_{9}\right) R^{1 / 4}$. Очевидно, найдется $R_{1} \in\left(0, R_{0}\right]$ такое, что $\left\|h_{R}^{+}-1\right\|<\|1\|$ при $0<R \leqslant R_{1}$. При этом $h_{R}^{+} \neq 0$, в частности пространство $H_{R}^{+}$нетривиально.

Пусть $R \in\left(0, R_{1}\right]$. Обозначим через $C_{R}^{+}$ограничение оператора $C_{R}$ на пространство $H_{R}^{+}$. Положим $D_{R}=1-I_{5}\left(1-C_{R}^{+}\right)$. Поскольку $\left\|C_{R}\right\| \leqslant 1-I_{1} R^{2}$, спектр $\sigma_{R}^{+}$оператора $C_{R}^{+}$лежит на отрезке $\left[1-R^{3 / 2}, 1-I_{1} R^{2}\right]\left(1-R^{3 / 2}<1-I_{1} R^{2}\right.$, так как $\left.I_{1} R_{0}^{1 / 2}<1\right)$. Тогда спектр оператора $D_{R}$ лежит на отрезке $\left[1-I_{5} R^{3 / 2}\right.$, $\left.1-I_{1} I_{5} R^{2}\right] \subset[0,1]$, в частности $\left\|D_{R}\right\| \leqslant 1-I_{1} I_{5} R^{2}$. Заметим, что

$$
\left\|D_{R} h_{R}^{+}-\left(1-I_{5} g R^{2}\right) h_{R}^{+}\right\|=I_{5}\left\|C_{R}^{+} h_{R}^{+}-\left(1-g R^{2}\right) h_{R}^{+}\right\| \leqslant I_{0} I_{5} R^{3} .
$$

Поскольку оператор умножения на функцию $g$ коммутирует с $C_{R}$ и оставляет инвариантным пространство $H_{R}^{+}$, он коммутирует с оператором $D_{R}$. Тогда

$$
\left\|D_{R}^{m} h_{R}^{+}-\left(1-I_{5} g R^{2}\right) D_{R}^{m-1} h_{R}^{+}\right\| \leqslant I_{0} I_{5} R^{3}\left\|D_{R}^{m-1}\right\|
$$

для любого натурального $m$, причем $\left\|D_{R}^{m-1}\right\|=\left\|D_{R}\right\|^{m-1}$, так как оператор $D_{R}$ самосопряжен. Ввиду неравенства $I_{0} I_{5} R_{0}^{2}<1$ функция $1-I_{5} g R^{2}$ положительна, в частности

$$
\sup \left|1-I_{5} g R^{2}\right|=1-I_{5} R^{2} \inf g \leqslant 1-I_{0}^{-1} I_{5} R^{2} .
$$

В итоге для любого натурального $n$ получаем:

$$
\begin{aligned}
\left\|D_{R}^{n} h_{R}^{+}-\left(1-I_{5} g R^{2}\right)^{n} h_{R}^{+}\right\| & \leqslant I_{0} I_{5} R^{3} \sum_{j=1}^{n}\left\|D_{R}\right\|^{j-1} \sup \left|1-I_{5} g R^{2}\right|^{n-j} \\
& \leqslant I_{0} I_{5} R^{3} \sum_{j=0}^{n-1}\left(1-I_{1} I_{5} R^{2}\right)^{j} \leqslant I_{0} I_{1}^{-1} R .
\end{aligned}
$$

Далее, оператор $p_{n}\left(C_{R}^{+}\right)-I_{4} D_{R}^{n}$ на пространстве $H_{R}^{+}$самосопряжен, а его спектр является образом спектра $\sigma_{R}^{+}$под действием многочлена $p_{n}(z)-I_{4}\left(1-I_{5}(1-z)\right)^{n}$. Поскольку $\sigma_{R}^{+} \subset\left[1-R^{3 / 2}, 1\right] \subset[1-\varepsilon, 1]$, из условия (W1b) следует, что спектр оператора $p_{n}\left(C_{R}^{+}\right)-I_{4} D_{R}^{n}$ лежит на отрезке $\left[-I_{6}\left(R^{3 / 2}+n R^{3}\right), I_{6}\left(R^{3 / 2}+n R^{3}\right)\right]$. При этом $\left\|p_{n}\left(C_{R}^{+}\right)-I_{4} D_{R}^{n}\right\| \leqslant I_{6}\left(R^{3 / 2}+n R^{3}\right)$. Тогда

$$
\left\|p_{n}\left(C_{R}^{+}\right) h_{R}^{+}-I_{4}\left(1-I_{5} g R^{2}\right)^{n} h_{R}^{+}\right\| \leqslant I_{0} I_{1}^{-1} I_{4} R+I_{6}\left(R^{3 / 2}+n R^{3}\right)\left\|h_{R}^{+}\right\|,
$$

причем $\left\|h_{R}^{+}\right\| \leqslant\left\|h_{R}\right\| \leqslant(2 \pi)^{1 / 2}+I_{0} R_{0}$. Таким образом,

$$
\left\|p_{n}\left(C_{R}^{+}\right) h_{R}^{+}-I_{4}\left(1-I_{5} g R^{2}\right)^{n} h_{R}^{+}\right\| \leqslant I_{10}\left(R+n R^{3}\right),
$$

где $I_{10}$ - положительная постоянная, не зависяшая от $R$ и $n$. Отсюда следует, что $\left\|p_{n}\left(C_{R}\right) 1-I_{4}\left(1-I_{5} g R^{2}\right)^{n}\right\| \leqslant I_{10}\left(R+n R^{3}\right)+\left(I_{4}+I_{7}\right)\left\|h_{R}^{+}-1\right\| \leqslant I_{11}\left(R^{1 / 4}+n R^{3}\right)$, где $I_{11}=\max \left(I_{10}, I_{10} R_{0}^{3 / 4}+\left(I_{4}+I_{7}\right)\left(I_{0} R_{0}^{3 / 4}+I_{9}\right)\right)$. Кроме того,

$$
\begin{aligned}
\left\|p_{n}\left(C_{R}\right) 1-I_{4}\left(1-I_{5} g R^{2}\right)^{n}\right\| & \leqslant(2 \pi)^{1 / 2}\left(\left\|p_{n}\left(C_{R}\right)\right\|+I_{4} \sup \left|1-I_{5} g R^{2}\right|^{n}\right) \\
& \leqslant I_{12} \exp \left(-I_{13} n R^{2}\right),
\end{aligned}
$$

где $I_{12}=(2 \pi)^{1 / 2}\left(I_{4}+I_{7}\right), I_{13}=\min \left(I_{8}, I_{0}^{-1} I_{5}\right)$. 
Для каждого натурального $n$ определим число $r_{n}>0$ из равенства $r_{n}=$ $\exp \left(-n r_{n}^{2}\right)$. Очевидно, $r_{n} \rightarrow 0$ при $n \rightarrow \infty$. Если $r_{n}<R_{1}$, то, как следует из полученных оценок,

$$
\begin{aligned}
& n \int_{0}^{R_{1}} R\left\|p_{n}\left(C_{R}\right) 1-I_{4}\left(1-I_{5} g R^{2}\right)^{n}\right\| d R \\
& \quad \leqslant n \int_{0}^{r_{n}} I_{11} R\left(R^{1 / 4}+n R^{3}\right) d R+n \int_{r_{n}}^{R_{1}} I_{12} R \exp \left(-I_{13} n R^{2}\right) d R \\
& \quad \leqslant \frac{4}{9} I_{11} n r_{n}^{9 / 4}+\frac{1}{5} I_{11} n^{2} r_{n}^{5}+\frac{1}{2} I_{12} I_{13}^{-1} \exp \left(-I_{13} n r_{n}^{2}\right) .
\end{aligned}
$$

Поскольку $n r_{n}^{2}=-\log r_{n}$, заключаем, что $n r_{n}^{2} \rightarrow \infty$, а $n r_{n}^{9 / 4} \rightarrow 0$ и $n^{2} r_{n}^{5} \rightarrow 0$ при $n \rightarrow \infty$. Тем самьм, последовательность функций

$$
n \int_{0}^{R_{1}} R\left(C_{R}^{(n)} 1-I_{4}\left(1-I_{5} g R^{2}\right)^{n}\right) d R, \quad n=1,2, \ldots
$$

стремится к нулю в пространстве $L_{2}\left(S^{1}\right)$. В то же время, последовательность функций

$$
n \int_{0}^{R_{1}} R\left(1-I_{5} g R^{2}\right)^{n} d R=\frac{n}{2(n+1) I_{5} g}\left(1-\left(1-I_{5} g R_{1}^{2}\right)^{n+1}\right), \quad n=1,2, \ldots
$$

при $n \rightarrow \infty$ равномерно сходится к функции $\left(2 I_{5} g\right)^{-1}$. Отсюда следует, что

$$
n \int_{0}^{R_{1}} R\left(C_{R}^{(n)} 1,1\right) d R \rightarrow\left(I_{4}\left(2 I_{5} g\right)^{-1}, 1\right), \quad n \rightarrow \infty,
$$

причем

$$
\left(I_{4}\left(2 I_{5} g\right)^{-1}, 1\right)=I_{4} \int_{S^{1}}\left(2 I_{5} g(t)\right)^{-1} d t \geqslant \pi I_{0}^{-1} I_{4} I_{5}^{-1}>0 .
$$

Свойство (Р1b) установлено.

ПРЕДЛОЖЕНИЕ 2.5. Пусть $\left\{C_{R}\right\}_{R>0}-$ семейство сжимающих операторов в $L_{2}\left(S^{1}\right)$, непрерывно зависящих от параметра $R$. Если это семейство обладает свойствами $\left(\mathrm{V}^{\prime}\right),(\mathrm{V} 1 \mathrm{~b})$ u $\left(\mathrm{V} 2^{\prime}\right)$, то семейство операторов $C_{R}^{(n)}=C_{R}^{n}$, $R>0, n=0,1, \ldots$, обладает свойствами $(\mathrm{P} 1),(\mathrm{P} 1 \mathrm{~b})$ u $(\mathrm{P} 2)$.

ДокАЗАТЕЛЬСтво. Поскольку оператор $C_{R}$ является сжимающим, $\left\|C_{R}\right\| \leqslant 1$, то для любого натурального $m$ и целого $n \geqslant 0$ имеет место оценка $\left\|C_{R}^{n}\right\| \leqslant$ $\left\|C_{R}^{m}\right\|^{\lfloor n / m\rfloor}$, где $\lfloor n / m\rfloor$ - целая часть числа $n / m$. Ввиду свойства (V1') можно выбрать $m$ так, что $\left\|C_{R}^{m}\right\| \leqslant 1-I_{1} R^{2}$ при $0<R \leqslant R_{0}$, где $I_{1}, R_{0}$ - положительные постоянные. Уменьшив, если нужно, постоянную $I_{1}$, добьемся того, что $1-I_{1} R_{0}^{2} \geqslant 1 / 2$. Тогда при $0<R \leqslant R_{0}$ имеет место оценка

$$
\left\|C_{R}^{n}\right\| \leqslant\left(1-I_{1} R^{2}\right)^{\lfloor n / m\rfloor} \leqslant 2\left(1-I_{1} R^{2}\right)^{n / m} \leqslant 2\left(1-m^{-1} I_{1} R^{2}\right)^{n} .
$$

Свойство (Р1) установлено. 
Ввиду свойства ( $\mathrm{V}^{\prime}$ ) для любых $R_{1}$ и $R_{2}, 0<R_{1}<R_{2}$, найдется натуральное $M$ такое, что $\left\|C_{R}^{M}\right\|<1$ при $R_{1} \leqslant R \leqslant R_{2}$. Функция $R \mapsto\left\|C_{R}^{M}\right\|$ непрерывна, так как оператор $C_{R}$ непрерьвно зависит от $R$, поэтому найдется $\rho \in(0,1)$ такое, что $\left\|C_{R}^{M}\right\| \leqslant \rho$ при $R_{1} \leqslant R \leqslant R_{2}$. Тогда для любого целого $n \geqslant 0$ при $R_{1} \leqslant R \leqslant R_{2}$ имеем

$$
\left\|C_{R}^{n}\right\| \leqslant\left\|C_{R}^{M}\right\|^{\lfloor n / M\rfloor} \leqslant \rho^{n / M-1}=\rho^{-1}\left(\rho^{1 / M}\right)^{n} .
$$

Свойство (Р2) установлено.

Воспользуемся свойством (V1b). Пусть $I_{0}$ и $R_{0}$ - постоянные, $R \in\left(0, R_{0}\right]$, а $g$ и $h_{R}$ - функции, фигурируюшие в формулировке этого свойства. Ввиду уже установленного свойства (P1) при малых значениях $R$ имеет место оценка $\left\|C_{R}^{n}\right\| \leqslant$ $I_{2}\left(1-I_{3} R^{2}\right)^{n}$, где $I_{2}, I_{3}$ - положительные постоянные. Уменьшив, если нужно, постоянную $R_{0}$, мы можем считать, что приведенная оценка верна при $0<R \leqslant R_{0}$, а также $R_{0}^{2} \max \left(I_{0}, I_{3}\right)<1$. Пусть $R \in\left(0, R_{0}\right]$. Тогда $0<1-I_{3} R^{2} \leqslant \exp \left(-I_{3} R^{2}\right)$, кроме того, $0<1-g R^{2} \leqslant 1-I_{0}^{-1} R^{2} \leqslant \exp \left(-I_{0}^{-1} R^{2}\right)$ на всей окружности. Поскольку оператор $C_{R}$ коммутирует с оператором умножения на функцию $g$, $\left\|C_{R}^{m} h_{R}-\left(1-g R^{2}\right) C_{R}^{m-1} h_{R}\right\| \leqslant I_{0} R^{3}\left\|C_{R}^{m-1}\right\|$ для любого натурального $m$. Тогда для любого натурального $n$ имеет место оценка

$\left\|C_{R}^{n} h_{R}-\left(1-g R^{2}\right)^{n} h_{R}\right\| \leqslant I_{0} R^{3} \sum_{j=1}^{n}\left\|C_{R}^{j-1}\right\| \leqslant I_{0} I_{2} R^{3} \sum_{j=0}^{n-1}\left(1-I_{3} R^{2}\right)^{j} \leqslant I_{0} I_{2} I_{3}^{-1} R$.

Как следствие, $\left\|C_{R}^{n} 1-\left(1-g R^{2}\right)^{n}\right\| \leqslant I_{4} R$, где $I_{4}=2 I_{0}+I_{0} I_{2} I_{3}^{-1}$. Кроме того, верна оценка

$$
\left\|C_{R}^{n} 1-\left(1-g R^{2}\right)^{n}\right\| \leqslant(2 \pi)^{1 / 2}\left(\left\|C_{R}^{n}\right\|+\sup \left|\left(1-g R^{2}\right)^{n}\right|\right) \leqslant I_{5} \exp \left(-I_{6} n R^{2}\right),
$$

где $I_{5}=(2 \pi)^{1 / 2}\left(I_{2}+1\right), I_{6}=\min \left(I_{3}, I_{0}^{-1}\right)$. Определим число $r_{n}>0$ из равенства $I_{4} r_{n}=I_{5} \exp \left(-I_{6} n r_{n}^{2}\right)$. Очевидно, $r_{n} \rightarrow 0$ при $n \rightarrow \infty$. Если $r_{n}<R_{0}$, то, как следует из полученных оценок,

$$
\begin{aligned}
& n\left\|\int_{0}^{R_{0}} R\left(C_{R}^{n} 1-\left(1-g R^{2}\right)^{n}\right) d R\right\| \leqslant n \int_{0}^{R_{0}} R\left\|C_{R}^{n} 1-\left(1-g R^{2}\right)^{n}\right\| d R \\
& \quad \leqslant n \int_{0}^{r_{n}} I_{4} R^{2} d R+n \int_{r_{n}}^{R_{0}} I_{5} R \exp \left(-I_{6} n R^{2}\right) d R \\
& \quad \leqslant \frac{1}{3} I_{4} n r_{n}^{3}+\frac{1}{2} I_{5} I_{6}^{-1} \exp \left(-I_{6} n r_{n}^{2}\right) .
\end{aligned}
$$

Поскольку $n r_{n}^{2}=-I_{6}^{-1} \log \left(I_{4} I_{5}^{-1} r_{n}\right)$, заключаем, что $n r_{n}^{2} \rightarrow \infty$, а $n r_{n}^{3} \rightarrow 0$ при $n \rightarrow \infty$. Тем самьм, последовательность функций

$$
n \int_{0}^{R_{0}} R\left(C_{R}^{n} 1-\left(1-g R^{2}\right)^{n}\right) d R, \quad n=1,2, \ldots,
$$

стремится к нулю в пространстве $L_{2}\left(S^{1}\right)$. В то же время, последовательность функций

$$
n \int_{0}^{R_{0}} R\left(1-g R^{2}\right)^{n} d R=\frac{n}{2(n+1) g}\left(1-\left(1-g R_{0}^{2}\right)^{n+1}\right), \quad n=1,2, \ldots,
$$


при $n \rightarrow \infty$ равномерно сходится к функции $(2 g)^{-1}$. Отсюда следует, что

$$
\begin{gathered}
n \int_{0}^{R_{0}} R\left(C_{R}^{n} 1,1\right) d R \rightarrow\left((2 g)^{-1}, 1\right), \quad n \rightarrow \infty, \\
\text { причем } \left.\left((2 g)^{-1}, 1\right)=\int_{S^{1}}(2 g(t))^{-1} d t \geqslant \pi I_{0}^{-1}>0 . \text { Свойство (Р } 1 \mathrm{~b}\right) \text { установлено. }
\end{gathered}
$$

\section{§3. Действия групп и полугрупп}

Нам понадобятся три леммы, доказанные в [5].

Лемма 3.1 [5]. Подгруппа $H$ группье $\mathbb{R}^{2}$ всюду плотна в последней тогда и лишь тогда, когда не существует ненулевого линейного функиионала на $\mathbb{R}^{2}$, который принимает иелое значение на каждом из элементов $H$.

ЛЕмма 3.2 [5]. Если угол $\varphi$ не кратен ни $\pi / 2$, ни $\pi / 3$, то группа, порожденная векторами $v_{n}=(\cos n \varphi, \sin n \varphi), n=0,1,2, \ldots$, всюду плотна в $\mathbb{R}^{2}$.

Лемма 3.3 [5]. Пусть $\mathscr{H}$ - подгруппа группь $\mathscr{G . ~ Е с л и ~ и з о м е т р и и ~ и з ~} \mathscr{H}$ не имеют общей неподвижной точки и не переводят в себя одну и ту же решетку вида $\mathscr{L}_{Q}$, где $Q$ - полоса, квадрат или правильный треугольник, то группа $\mathscr{H} \cap \mathscr{G}_{0}$ всюду плотна в $\mathscr{G}_{0}$.

Добавим к ним еще две леммы о свойствах подполугрупп групшы $\mathscr{G}$.

Лемма 3.4. Пусть $\mathscr{H}$ - полугруппа изометрий плоскости Р. Если $\mathscr{H}$ содержит хотя бы один поворот на ненулевой угол, но не содержит поворотов на угль, несоизмеримые с $\pi$, то полугруппа Н Я явлется группой.

ДокАЗАТЕльство. Нужно показать, что для любой изометрии $A \in \mathscr{H}$ обратное преобразование $A^{-1}$ также принадлежит полугруппе $\mathscr{H}$. Это очевидно, если изометрия $A$ имеет конечный порядок. Таковы осевые симметрии, а также, как следует из условия леммы, все содержашиеся в $\mathscr{H}$ повороты. Далее, согласно условию в $\mathscr{H}$ найдется поворот $B$ на ненулевой угол. Этот поворот имеет конечнњй порядок $m>1$. Для любого параллельного переноса $A \in \mathscr{H}$ изометрия $A B$ является поворотом плоскости на тот же угол, что и $B$ (но вокруг другой точки), в частности $A B$ имеет порядок $m$. Как следствие, $A^{-1}=B(A B)^{m-1} \in \mathscr{H}$. Наконец, если $A \in \mathscr{H}$ - скользящая симметрия, то $A^{2}$ - параллельный перенос. Как уже показано, полугруппа $\mathscr{H}$ содержит изометрию $\left(A^{2}\right)^{-1}$, а тем самым, и изометрию $\left(A^{2}\right)^{-1} A=A^{-1}$.

ЛЕмма 3.5. Пусть $\mathscr{H}$ - полугруппа, порожсденная изометриями

$$
A_{1}, \ldots, A_{k} \in \mathscr{G}
$$

Если полугруппа $\mathscr{H}$ не содержит поворотов и не является группой, то изометрии $A_{1}, \ldots, A_{k}$ удовлетворяют одному из условий (i), (ii) теоремы 1.4. 
ДокАЗАТЕЛЬСтво. Пусть $P_{0}$ - векторное евклидово пространство, ассоциированное с афффинной плоскостью $P$. Параллельный перенос плоскости $P$ на вектор $v \in P_{0}$ обозначим $T_{v}$. Вначале предположим, что каждая из изометрий $A_{j}$, $1 \leqslant j \leqslant k$, имеет вид $T_{v_{j}}$, где $v_{j} \in P_{0}$. Если сумма векторов $v_{1}, \ldots, v_{k}$ ненулевая, то изометрии $A_{1}, \ldots, A_{k}$ удовлетворяют условию (i) теоремы 1.4. В противном случае $A_{1} A_{2} \ldots A_{k}$ - тождественное преобразование. Отсюда следует, что изометрии $A_{1}, \ldots, A_{k}$ обратимы в полугруппе $\mathscr{H}$. Тогда любой элемент полугруппы $\mathscr{H}$ обратим в ней, т.е. $\mathscr{H}$ - группа.

Теперь рассмотрим случай, когда среди изометрий $A_{1}, \ldots, A_{k}$ есть изометрии, не принадлежащие $\mathscr{G}_{0}$. Пусть, для определенности, таковы изометрии $A_{1}, \ldots, A_{k_{1}}$, $1 \leqslant k_{1} \leqslant k$. Поскольку полугруппа $\mathscr{H}$ не содержит поворотов, все они суть осевые или скользящие симметрии, оси которых параллельны некоторой фиксированной прямой $l$. Пусть $S$ - осевая симметрия относительно прямой $l$. Возьмем произвольную точку $x \in l$. Для любого $j, 1 \leqslant j \leqslant k$, обозначим через $v_{j}$ вектор $A_{j} x-x \in P_{0}$. Тогда $A_{j}=T_{v_{j}} S$ при $1 \leqslant j \leqslant k_{1}$ и $A_{j}=T_{v_{j}}$ при $k_{1}<j \leqslant k$. Преобразование $A_{1}^{2} A_{2}^{2} \ldots A_{k}^{2}$ является параллельньм переносом на вектор

$$
w=\sum_{1 \leqslant j \leqslant k_{1}}\left(v_{j}+S v_{j}\right)+2 \sum_{k_{1}<j \leqslant k} v_{j}
$$

где $S v_{j}$ обозначает вектор, симметричньй $v_{j}$ относительно прямой $l$. Ортогональная проекция вектора $w / 2$ на направление прямой $l$ такая же, как и у суммы векторов $v_{1}, \ldots, v_{k}$. Тем самым, если вектор $w$ не ортогонален прямой $l$, то изометрии $A_{1}, \ldots, A_{k}$ удовлетворяют условию (ii) теоремы 1.4 . Если же вектор $w$ ортогонален $l$, то $S T_{w}=T_{-w} S$, тогда $T_{w} A_{1} T_{w}=A_{1}$. Как следствие, $A_{1} A_{2}^{2} \ldots A_{k}^{2} A_{1}^{3} A_{2}^{2} \ldots A_{k}^{2}$ - тождественное преобразование. Таким образом, полугруппа $\mathscr{H}$ содержит изометрию $A_{k}^{-2} \ldots A_{2}^{-2} A_{1}^{-3} A_{k}^{-2} \ldots A_{2}^{-2} A_{1}^{-1}$. Тогда она содержит изометрии $A_{1}^{-1}, \ldots, A_{k}^{-1}$, откуда следует, что $\mathscr{H}$ - группа.

Теперь разберем вырожденные случаи (i) и (ii) теоремы 1.4 .

Лемма 3.6. Если изометрии $A_{1}, \ldots, A_{k} \in \mathscr{G}$ удовлетворяют одному из условий (i), (ii) теоремы 1.4 , то действие $d=\mathrm{FSG}_{k}\left[A_{1}, \ldots, A_{k}\right]$ удовлетворяет условию 2) той же теоремь.

ДокАЗАТЕЛЬСТво. Вначале докажем вспомогательное комбинаторное утверждение. Пусть $a_{1}, \ldots, a_{k}$ - образующие полугруппы $\mathrm{FSG}_{k}$. Произвольный элемент $g \in \mathrm{FSG}_{k}$ длины $n>0$ имеет единственное представление вида $a_{j_{1}} a_{j_{2}} \cdots a_{j_{n}}$, где $1 \leqslant j_{s} \leqslant k, s=1, \ldots, n$. Для каждого $j \in\{1, \ldots, k\}$ обозначим через $m_{j}(g)$ число элементов последовательности $j_{1}, \ldots, j_{n}$, равных $j$. Пусть $\varepsilon \in\left(0, k^{-1}\right)$. Обозначим через $\widetilde{N}_{\varepsilon, j}^{(n)}, 1 \leqslant j \leqslant k$, отношение числа элементов $g$ длины $n$, для которых $m_{j}(g) \leqslant\left(k^{-1}-\varepsilon\right) n$, к числу $k^{n}$ всех элементов длины $n$ в $\mathrm{FSG}_{k}$. Через $\widetilde{N}_{\varepsilon}^{(n)}$ обозначим отношение к $k^{n}$ числа тех элементов $g \in \mathrm{FSG}_{k}$ длины $n$, для которых хотя бы одно из чисел $m_{1}(g), \ldots, m_{k}(g)$ не превосходит $\left(k^{-1}-\varepsilon\right) n$. Очевидно, величины $\widetilde{N}_{\varepsilon, 1}^{(n)}, \ldots, \widetilde{N}_{\varepsilon, k}^{(n)}$ равны и $\widetilde{N}_{\varepsilon}^{(n)} \leqslant k \widetilde{N}_{\varepsilon, 1}^{(n)}$. Как нетрудно увидеть,

$$
\tilde{N}_{\varepsilon, 1}^{(n)}=k^{-n} \sum_{0 \leqslant j \leqslant\left(k^{-1}-\varepsilon\right) n}\left(\begin{array}{l}
n \\
j
\end{array}\right)(k-1)^{n-j},
$$


здесь $\left(\begin{array}{c}n \\ j\end{array}\right)=\frac{n !}{j !(n-j) !}-$ биномиальный коэффициент. Заметим, что неравенство $j \leqslant\left(k^{-1}-\varepsilon\right) n$ влечет неравенство $(k-1+k \varepsilon) j \leqslant(1-k \varepsilon)(n-j)$. Как следствие, для любого $\delta>0$ имеет место оценка

$$
\begin{aligned}
\tilde{N}_{\varepsilon, 1}^{(n)} & \leqslant k^{-n} \sum_{0 \leqslant j \leqslant\left(k^{-1}-\varepsilon\right) n}\left(\begin{array}{c}
n \\
j
\end{array}\right) e^{-\delta(k-1+k \varepsilon) j} e^{\delta(1-k \varepsilon)(n-j)}(k-1)^{n-j} \\
& \leqslant k^{-n}\left(e^{-\delta(k-1+k \varepsilon)}+(k-1) e^{\delta(1-k \varepsilon)}\right)^{n}
\end{aligned}
$$

Если $\delta$ достаточно мало, то число $e^{-\delta(k-1+k \varepsilon)}+(k-1) e^{\delta(1-k \varepsilon)}$ меньше $k$. Тем самьп, найдется $I_{\varepsilon}>0$ такое, что последовательность $\left\{\exp \left(I_{\varepsilon} n\right) \widetilde{N}_{\varepsilon, 1}^{(n)}\right\}$ ограничена. При этом последовательность $\left\{\exp \left(I_{\varepsilon} n\right) \widetilde{N}_{\varepsilon}^{(n)}\right\}$ также ограничена.

Пусть $A_{1}, \ldots, A_{k}$ - изометрии, удовлетворяющие условию (i). Все они суть параллельные переносы на некоторые векторы $v_{1}, \ldots, v_{k}$, причем сумма $v=v_{1}+$ $\cdots+v_{k}$ ненулевая. Выберем $\varepsilon \in\left(0, k^{-1}\right)$ такое, что $2 k^{2} \varepsilon\left(\left|v_{1}\right|+\cdots+\left|v_{k}\right|\right) \leqslant|v|$. Пусть $x \in P$, а $E$ - ограниченное подмножество $P$. Выберем натуральное $n_{0}$ такое, что расстояние от точки $x$ до любой точки множества $E$ не больше $n_{0}|v| /(2 k)$. Пусть $g$ - произвольный элемент $\mathrm{FSG}_{k}$ длины $n \geqslant n_{0}$. Изометрия $d(g)$ является параллельным переносом на вектор $w_{g}=m_{1}(g) v_{1}+\cdots+m_{k}(g) v_{k}$. Предположим, что $m_{j}(g)>\left(k^{-1}-\varepsilon\right) n$ для любого $j, 1 \leqslant j \leqslant k$. Поскольку $m_{1}(g)+$ $\cdots+m_{k}(g)=n$, отсюда следует, что $\left|m_{j}(g)-n / k\right|<k \varepsilon n, j=1, \ldots, k$. Тогда $\left|w_{g}-(n / k) v\right|<k \varepsilon n\left(\left|v_{1}\right|+\cdots+\left|v_{k}\right|\right) \leqslant n|v| /(2 k)$. При этом $\left|w_{g}\right|>n|v| /(2 k)$, в частности $d(g) x \notin E$. Таким образом, $d(g) x \in E$ лишь в том случае, когда хотя бы одно из чисел $m_{1}(g), \ldots, m_{k}(g)$ не превосходит $\left(k^{-1}-\varepsilon\right) n$. Отсюда следует, что $N_{d, x}^{(n)}(E) \leqslant \tilde{N}_{\varepsilon}^{(n)}$ при $n \geqslant n_{0}$. Тогда последовательность $\left\{\exp \left(I_{\varepsilon} n\right) N_{d, x}^{(n)}(E)\right\}$ ограничена.

Теперь предположим, что изометрии $A_{1}, \ldots, A_{k}$ удовлетворяют условию (ii). При этом каждая из них является либо параллельньм переносом, либо осевой или скользящей симметрией, ось которой параллельна некоторой фиксированной прямой $l$. Выберем точку $x \in P$. Для любого $j, 1 \leqslant j \leqslant k$, обозначим через $w_{j}$ ортогональную проекцию вектора $A_{j} x-x$ на направление прямой $l$. Заметим, что векторы $w_{1}, \ldots, w_{k}$ не зависят от выбора точки $x$. Ввиду условия (ii) их сумма ненулевая. Положим $d_{1}=\mathrm{FSG}_{k}\left[B_{1}, \ldots, B_{k}\right]$, где $B_{1}, \ldots, B_{k}$ - параллельные переносы на векторы $w_{1}, \ldots, w_{k}$ соответственно. Изометрии $B_{1}, \ldots, B_{k}$ удовлетворяют условию (i), поэтому, как уже доказано, найдется $I_{0}>0$ такое, что для любого ограниченного множества $E_{0} \subset P$ и любой точки $x_{0} \in P$ последовательность $\left\{\exp \left(I_{0} n\right) N_{d_{1}, x_{0}}^{(n)}\left(E_{0}\right)\right\}$ ограничена. Пусть $x \in P$, а $E$ - ограниченное подмножество $P$. Обозначим через $E_{1}$ ортогональную проекцию множества $E$ на прямую $l$, а через $x_{1}$ - ортогональную проекцию точки $x$ на $l$. Легко видеть, что для любого $g \in \mathrm{FSG}_{k}$ точка $d_{1}(g) x_{1}$ является ортогональной проекцией точки $d(g) x$ на прямую $l$. Тем самым, $d_{1}(g) x_{1} \in E_{1}$, как только $d(g) x \in E$. Отсюда следует, что $N_{d, x}^{(n)}(E) \leqslant N_{d_{1}, x_{1}}^{(n)}\left(E_{1}\right)$ при $n=0,1, \ldots$ Множество $E_{1}$ ограничено, так как ограничено множество $E$. Тогда последовательность $\left\{\exp \left(I_{0} n\right) N_{d_{1}, x_{1}}^{(n)}\left(E_{1}\right)\right\}$ ограничена, а с ней ограничена и последовательность $\left\{\exp \left(I_{0} n\right) N_{d, x}^{(n)}(E)\right\}$. 
Пусть $A_{1}, \ldots, A_{k} \in \mathscr{G}$. Обозначим через $\mathscr{H}$ полугруппу, порожденную изометриями $A_{1}, \ldots, A_{k}$. Положим $C=k^{-1}\left(u\left[A_{1}\right]+\cdots+u\left[A_{k}\right]\right)$. Пусть $\left\{C_{R}\right\}_{R>0}$ - семейство радиальных операторов, соответствующих оператору $C$ в некоторой декартовой системе координат. Следующие три леммы определяют условия, при которых это семейство обладает свойствами $\left(\mathrm{V} 1^{\prime}\right),(\mathrm{V} 1 \mathrm{~b})$ и $\left(\mathrm{V} 2^{\prime}\right)$, сформулированньгии в $\S 2$.

Лемма 3.7. Если изометрии $A_{1}, \ldots, A_{k}$ не имеют общей неподвижной точки, а полугруппа $\mathscr{H}$ содержит поворот на угол, не кратный ни $\pi / 2$, ни $\pi / 3$, то семейство операторов $C_{R}, R>0$, обладает свойствами (V1') $u\left(\mathrm{~V} 2^{\prime}\right)$.

ДоказАтеЛЬСтво. Пусть $A \in \mathscr{H}$ - поворот на угол, не кратный ни $\pi / 2$, ни $\pi / 3$. Пусть $x$ - центр поворота $A$. Покажем, что полугруппа $\mathscr{H}$ содержит поворот $B$, не коммутирующий с $A$, т.е. имеющий другой центр. Если $\mathscr{H}$ содержит нетривиальный параллельный перенос $T$, то можно положить $B=T A$. Если в $\mathscr{H}$ имеется осевая симметрия, ось которой не проходит через точку $x$, то можно положить $B=S A S$. Отсюда следует, что если в полугруппе $\mathscr{H}$ нет требуемого поворота, то она состоит из поворотов и осевых симметрий, переводящих в себя точку $x$. Последнее противоречит условию леммы.

Пусть $d=\mathrm{FSG}_{k}\left[A_{1}, \ldots, A_{k}\right]$. Поскольку полугруппа $\mathscr{H}$ содержит повороты $A$ и $B$, найдутся элементы $g_{1}, g_{2} \in \mathrm{FSG}_{k}$ такие, что $d\left(g_{1}\right)=A, d\left(g_{2}\right)=B$. Положим $m_{1}=\left|g_{1}\right|, m_{2}=\left|g_{2}\right|$. Пусть $n$ - натуральное число, которое не больше порядка поворота $A$. Изометрии $A, A^{2}, \ldots, A^{n-1}$ суть нетривиальные повороты вокруг точки $x$, поэтому ни одна из них не коммутирует с поворотом $B$. Отсюда следует, что изометрии $A^{j} B A^{n-1-j}, j=0,1, \ldots, n-1$, попарно различны. Элементы $g_{1}^{j} g_{2} g_{1}^{n-1-j}, j=0,1, \ldots, n-1$, полугруппы $\mathrm{FSG}_{k}$ имеют одну и ту же длину $m=(n-1) m_{1}+m_{2}$ и различны, так как $d\left(g_{1}^{j} g_{2} g_{1}^{n-1-j}\right)=$ $A^{j} B A^{n-1-j}$. Тем самым, оператор $(k C)^{m}=\sum_{|g|=m} u[d(g)]$ имеет вид $n D+Y$, где $D=n^{-1} \sum_{j=0}^{n-1} u\left[A^{j} B A^{n-1-j}\right]$, а $Y$ - сумма $k^{m}-n$ операторов вида $u[X]$, $X \in \mathscr{G}$. Согласно лемме $2.2\left(k C_{R}\right)^{m}=n D_{R}+Y_{R}$ для любого $R>0$, причем $Y_{R}$ - сумма $k^{m}-n$ унитарных операторов в $L_{2}\left(S^{1}\right)$. Как следствие, $1-\left\|C_{R}^{m}\right\| \geqslant$ $n k^{-m}\left(1-\left\|D_{R}\right\|\right)$. Поворот $B$ можно представить в виде $T_{0} B_{0}$, где $T_{0}-$ нетривиальный параллельный перенос, а $B_{0}$ - поворот вокруг точки $x$. При этом $n D=$ $u\left[A^{n-1} B_{0}\right] \sum_{j=0}^{n-1} u\left[A^{j} T_{0} A^{-j}\right]$. Относительно декартовых координат, в которых вычисляются радиальные операторы, $T_{0}$ есть параллельньй перенос на ненулевой вектор $v \in \mathbb{R}^{2}$. При этом изометрия $A^{j} T_{0} A^{-j}$ есть параллельный перенос на вектор $A_{0}^{j} v$, где $A_{0}$ - линейно однородная часть поворота $A$. Тогда для любого $R>0$ радиальный оператор $u\left[A^{n-1} B_{0}\right]_{R}^{-1} D_{R}=n^{-1} \sum_{j=0}^{n-1} u\left[A^{j} T_{0} A^{-j}\right]_{R}$ есть оператор умножения на функцию $h_{n, R}=n^{-1} \sum_{j=0}^{n-1} \exp \left(i R\left(\Phi, A_{0}^{j} v\right)\right)$. Поскольку оператор $u\left[A^{n-1} B_{0}\right]_{R}$ унитарен, $\left\|D_{R}\right\|=\sup \left|h_{n, R}\right|$. В итоге, $1-\left\|C_{R}^{m}\right\| \geqslant$ $n k^{-m}\left(1-\sup \left|h_{n, R}\right|\right)$.

Выберем $I_{1}>0$ такое, что $\left|e^{i y}-\left(1+i y-y^{2} / 2\right)\right| \leqslant I_{1}|y|^{3}$ при $|y| \leqslant 1$. Тогда при $0<R \leqslant|v|^{-1}$ для любого натурального $n$ имеет место оценка

$$
\sup \left|h_{n, R}-\left(1+i R h_{n}^{(1)}-R^{2} h_{n}^{(2)}\right)\right| \leqslant I_{1}|v|^{3} R^{3},
$$


где

$$
h_{n}^{(1)}=\frac{1}{n} \sum_{j=0}^{n-1}\left(\Phi, A_{0}^{j} v\right), \quad h_{n}^{(2)}=\frac{1}{2 n} \sum_{j=0}^{n-1}\left(\Phi, A_{0}^{j} v\right)^{2} .
$$

Положим $I_{0}=\inf \left((\Phi, v)^{2}+\left(\Phi, A_{0} v\right)^{2}\right)$. Векторы $v$ и $A_{0} v$ неколлинеарны, поэтому скалярные произведения $(\Phi(t), v)$ и $\left(\Phi(t), A_{0} v\right)$ не равны нулю одновременно. Учитывая непрерывность отображения $\Phi$, заключаем, что $I_{0}>0$. Для любого $t \in S^{1}$ и целого $j \geqslant 0$ верно равенство $\left(\Phi(t), A_{0}^{j} v\right)=(\Phi(t-j \varphi), v)$, где $\varphi-$ угол поворота $A$. Отсюда следует, что $\inf \left(\left(\Phi, A_{0}^{j} v\right)^{2}+\left(\Phi, A_{0}^{j+1} v\right)^{2}\right)=I_{0}$ при $j=0,1, \ldots$ Таким образом, если $n \geqslant 2$, то

$$
\inf \sum_{j=0}^{n-1}\left(\Phi, A_{0}^{j} v\right)^{2} \geqslant \frac{(n-1) I_{0}}{2} \geqslant \frac{n I_{0}}{4}
$$

т.е. $\inf h_{n}^{(2)} \geqslant I_{0} / 8$. Заметим, что отношение длин векторов $v_{n}=\sum_{j=0}^{n-1} A_{0}^{j} v$ и $v$ равно $\left|1+e^{i \varphi}+\cdots+e^{i(n-1) \varphi}\right|=\left|1-e^{i n \varphi}\right| /\left|1-e^{i \varphi}\right|$, тем самьм, в последовательности $v_{1}, v_{2}, \ldots$ есть векторы сколь угодно малой длины. Если порядок поворота $A$ конечен, положим $n$ равным этому порядку, при этом $v_{n}=0$. Если порядок $A$ бесконечен, выберем $n \geqslant 2$ такое, что $\left|v_{n}\right| \leqslant I_{0}^{1 / 2} / 4$. В любом случае $\sup \left|h_{n}^{(1)}\right|=n^{-1}\left|v_{n}\right| \leqslant I_{0}^{1 / 2} / 4$. Далее, $\inf h_{n}^{(2)} \geqslant I_{0} / 8$, так как $n \geqslant 2$. Наконец, $n$ не больше порядка поворота $A$, поэтому найдутся натуральное число $m$ и постоянная $I_{2}>0$ такие, что $1-\left\|C_{R}^{m}\right\| \geqslant I_{2}\left(1-\sup \left|h_{n, R}\right|\right)$ для любого $R>0$. Возьмем произвольное $R \in\left(0,|v|^{-1}\right)$. Поскольку $\sup h_{n}^{(2)} \leqslant|v|^{2} / 2$, функция $1-R^{2} h_{n}^{(2)}$ положительна. При этом

$$
\sup \left|1-R^{2} h_{n}^{(2)}\right|^{2} \leqslant \sup \left|1-R^{2} h_{n}^{(2)}\right|=1-R^{2} \inf h_{n}^{(2)} \leqslant 1-\frac{I_{0} R^{2}}{8} .
$$

Поскольку функции $h_{n}^{(1)}$ и $h_{n}^{(2)}$ вещественнозначны,

$$
\sup \left|1+i R h_{n}^{(1)}-R^{2} h_{n}^{(2)}\right|^{2}=\sup \left|1-R^{2} h_{n}^{(2)}\right|^{2}+R^{2} \sup \left|h_{n}^{(1)}\right|^{2} \leqslant 1-\frac{I_{0} R^{2}}{16}
$$

тогда $\sup \left|1+i R h_{n}^{(1)}-R^{2} h_{n}^{(2)}\right| \leqslant 1-I_{0} R^{2} / 32$. Наконец,

$$
\sup \left|h_{n, R}\right| \leqslant \sup \left|1+i R h_{n}^{(1)}-R^{2} h_{n}^{(2)}\right|+I_{1}|v|^{3} R^{3} \leqslant 1-\frac{I_{0} R^{2}}{32}+I_{1}|v|^{3} R^{3} .
$$

Отсюда вытекает, что при достаточно малых значениях $R$ имеет место оценка $\sup \left|h_{n, R}\right| \leqslant 1-I_{0} R^{2} / 64$; при этом $\left\|C_{R}^{m}\right\| \leqslant 1-I_{0} I_{2} R^{2} / 64$. Свойство (V1') установлено.

Выберем произвольное $R_{1}>0$. Векторы $A_{0} v-v$ и $A_{0}\left(A_{0} v-v\right)$ неколлинеарны, поэтому каждый линейньй функционал на $\mathbb{R}^{2}$, принимающий на них целые значения, является целочисленной линейной комбинацией функционалов $l_{0}$ и $l_{1}$, заданных соотношениями $l_{0}\left(A_{0} v-v\right)=l_{1}\left(A_{0}\left(A_{0} v-v\right)\right)=1, l_{0}\left(A_{0}\left(A_{0} v-v\right)\right)=$ $l_{1}\left(A_{0} v-v\right)=0$. Лишь конечное число таких функционалов имеет норму, не превышаюшую $(2 \pi)^{-1} R_{1}$. Поскольку $A$ есть поворот на угол, не кратный ни $\pi / 2$, ни $\pi / 3$, 
из леммы 3.2 следует, что векторы $A_{0}^{j+1} v-A_{0}^{j} v=A_{0}^{j}\left(A_{0} v-v\right), j=0,1, \ldots$, порождают подгруппу группы $\mathbb{R}^{2}$, всюду плотную в $\mathbb{R}^{2}$. Тогда из леммы 3.1 следует, что произвольный ненулевой линейный функционал на пространстве $\mathbb{R}^{2}$ принимает нецелое значение на некотором векторе $A_{0}^{j+1} v-A_{0}^{j} v, j \geqslant 0$. Тем самым, найдется натуральное $n$ такое, что каждый ненулевой линейный функционал на $\mathbb{R}^{2}$, норма которого не больше $(2 \pi)^{-1} R_{1}$, принимает нецелое значение хотя бы на одном из $n-1$ векторов $A_{0}^{j+1} v-A_{0}^{j} v, j=0,1, \ldots, n-2$. Если поворот $A$ имеет конечный порядок $n_{0}$, то $n$ можно выбрать не превышающим $n_{0}+1$, так как тогда $A_{0}^{n_{0}+j+1} v-A_{0}^{n_{0}+j} v=A_{0}^{j+1} v-A_{0}^{j} v$ для любого целого $j \geqslant 0$. Более того, $n$ можно выбрать не превьшаюшим $n_{0}$, так как сумма векторов $A_{0}^{j+1} v-A_{0}^{j} v$, $j=0,1, \ldots, n_{0}-1$, равна нулю. При этом, как показано выше, найдутся натуральное число $M$ и постоянная $I_{3}>0$ такие, что для любого $R>0$ имеет место неравенство $1-\left\|C_{R}^{M}\right\| \geqslant I_{3}\left(1-\sup \left|h_{n, R}\right|\right)$. Если $\left|h_{n, R}(t)\right|=1$ для некоторого $t \in S^{1}$, то числа $\exp \left(i R\left(\Phi(t), A_{0}^{j} v\right)\right), j=0,1, \ldots, n-1$, равны. Тогда числа $(2 \pi)^{-1} R\left(\Phi(t), A_{0}^{j+1} v-A_{0}^{j} v\right), j=0,1, \ldots, n-2$, являются целыми. Отображение $w \mapsto(2 \pi)^{-1} R(\Phi(t), w)$ есть ненулевой линейный функционал на $\mathbb{R}^{2}$, норма которого равна $(2 \pi)^{-1} R$. Тем самым, при $0<R \leqslant R_{1}$ имеем $\left|h_{n, R}\right|<1$ на всей окружности. Поскольку функция $h_{n, R}$ непрерьвна, $\sup \left|h_{n, R}\right|<1$ при $0<R \leqslant R_{1}$. Как следствие, $\left\|C_{R}^{M}\right\|<1$ при $0<R \leqslant R_{1}$. Ввиду произвольности выбора $R_{1}$ семейство операторов $\left\{C_{R}\right\}_{R>0}$ обладает свойством $\left(\mathrm{V} 2^{\prime}\right)$.

ЛЕмма 3.8. Если изометрии $A_{1}, \ldots, A_{k}$ не имеют общей неподвижсной точки и не переводят в себя одну и ту же решетку вида $\mathscr{L}_{Q}$, әде $Q-$ полоса, квадрат или правильный треугольник, а полугруппа Н Является группой, то семейство операторов $C_{R}, R>0$, обладает свойствами $\left(\mathrm{V} 1^{\prime}\right)$ u $\left(\mathrm{V} 2^{\prime}\right)$.

ДокАЗАТЕльство. Пусть $d=\mathrm{FSG}_{k}\left[A_{1}, \ldots, A_{k}\right]$. Поскольку полугруппа $\mathscr{H}$ является группой, найдется элемент $g_{0} \in \mathrm{FSG}_{k}$ положительной длины $m_{0}$ такой, что $d\left(g_{0}\right)$ - тождественное преобразование.

Декартовы координаты, в которых вычислялись радиальные операторы $C_{R}$, $R>0$, позволяют рассматривать произвольный элемент групшы $\mathscr{G}_{0}$ как параллельный перенос на некоторьй вектор $v \in \mathbb{R}^{2}$; мы обозначаем этот элемент $T_{v}$. Положим $H_{0}=\left\{v \in \mathbb{R}^{2} \mid T_{v} \in \mathscr{H}\right\}$. Через $H_{1}$ обозначим множество векторов $v \in \mathbb{R}^{2}$ таких, что каждый из параллельных переносов $T_{v}$ и $T_{-v}$ можно представить в виде $d(g)$, где $g$ - элемент полугруппы $\mathrm{FSG}_{k}$, длина $|g|$ которого делится на $m_{0}$. Множество $H_{0}$ является подгруппой $\mathbb{R}^{2}$, так как $\mathscr{H}$ - группа. Множество $H_{1}$ также является подгруппой $\mathbb{R}^{2}$, что следует непосредственно из его определения. Согласно лемме 3.3 группа $\mathscr{H} \cap \mathscr{G}_{0}$ всюду плотна в $\mathscr{G}_{0}$, откуда следует, что группа $H_{0}$ всюду плотна в $\mathbb{R}^{2}$. Группа $H_{1}$ также всюду плотна в $\mathbb{R}^{2}$, поскольку она содержит групшу $m_{0} H_{0}=\left\{m_{0} v \mid v \in H_{0}\right\}$.

Пусть векторы $v_{1}, \ldots, v_{n} \in H_{1}$ таковы, что среди векторов $v_{1},-v_{1}, \ldots, v_{n},-v_{n}$ нет двух одинаковых. Согласно определению множества $H_{1}$ найдутся элементы $g_{1}^{+}, g_{1}^{-}, \ldots, g_{n}^{+}, g_{n}^{-}$полугруппы $\mathrm{FSG}_{k}$, длины которых делятся на $m_{0}$, такие, что $d\left(g_{j}^{+}\right)=T_{v_{j}}, d\left(g_{j}^{-}\right)=T_{-v_{j}}, j=1, \ldots, n$. Поскольку $d\left(g_{0}^{p} g\right)=d(g)$ для любого $g \in \mathrm{FSG}_{k}$ и натурального $p$, причем $\left|g_{0}^{p} g\right|=p m_{0}+|g|$, элементы $g_{1}^{+}, g_{1}^{-}, \ldots, g_{n}^{+}, g_{n}^{-}$ можно выбрать таким образом, чтобы все они имели одну и ту же длину $m>0$. 
При этом все выбранные элементы различны, так как различны параллельные переносы $T_{v_{1}}, T_{-v_{1}}, \ldots, T_{v_{n}}, T_{-v_{n}}$. Отсюда следует, что оператор

$$
(k C)^{m}=\sum_{|g|=m} u[d(g)]
$$

имеет вид $2 n D+Y$, где $D=(2 n)^{-1}\left(u\left[T_{v_{1}}\right]+u\left[T_{-v_{1}}\right]+\cdots+u\left[T_{v_{n}}\right]+u\left[T_{-v_{n}}\right]\right)$, а $Y$ - сумма $k^{m}-2 n$ операторов вида $u[A], A \in \mathscr{G}$. Согласно лемме $2.2\left(k C_{R}\right)^{m}=$ $2 n D_{R}+Y_{R}$ для любого $R>0$, причем $Y_{R}$ - сумма $k^{m}-2 n$ унитарных операторов в $L_{2}\left(S^{1}\right)$. Как следствие, $1-\left\|C_{R}^{m}\right\| \geqslant 2 n k^{-m}\left(1-\left\|D_{R}\right\|\right)$. Из леммы 2.2 вытекает, что оператор $D_{R}$ есть оператор умножения на функцию $h_{R}=$ $n^{-1} \sum_{j=1}^{n} \cos \left(R\left(\Phi, v_{j}\right)\right)$. При этом $\left\|D_{R}\right\|=\sup \left|h_{R}\right|$.

Поскольку группа $H_{1}$ плотна в $\mathbb{R}^{2}$, она содержит пару неколлинеарных векторов $v_{1}$ и $v_{2}$. При этом векторы $v_{1},-v_{1}, v_{2},-v_{2}$ попарно различны. Из сказанного выше следует, что найдутся натуральное число $m$ и постоянная $I_{1}>0$ такие, что для любого $R>0$ имеет место оценка $\left\|C_{R}^{m}\right\| \leqslant 1-I_{1}\left(1-\sup \left|h_{R}\right|\right)$, где $h_{R}=\left(\cos \left(R\left(\Phi, v_{1}\right)\right)+\cos \left(R\left(\Phi, v_{2}\right)\right)\right) / 2$. Выберем постоянную $R_{0}>0$ такую, что $R_{0}\left|v_{1}\right| \leqslant \pi / 2$ и $R_{0}\left|v_{2}\right| \leqslant \pi / 2$. При $0<R \leqslant R_{0}$ на всей окружности $S^{1}$ имеем $0 \leqslant h_{R} \leqslant 1-\frac{1}{4} R^{2}\left(\left(\Phi, v_{1}\right)^{2}+\left(\Phi, v_{2}\right)^{2}\right)$. Тогда $1-\sup \left|h_{R}\right| \geqslant I_{0} R^{2} / 4$, где $I_{0}=\inf \left(\left(\Phi, v_{1}\right)^{2}+\left(\Phi, v_{2}\right)^{2}\right)$. Поскольку векторы $v_{1}$ и $v_{2}$ неколлинеарны, скалярные произведения $\left(\Phi(t), v_{1}\right)$ и $\left(\Phi(t), v_{2}\right)$ не равны нулю одновременно. Как следствие, постоянная $I_{0}$ положительна. При этом семейство $\left\{C_{R}\right\}_{R>0}$ обладает свойством $\left(\mathrm{V} 1^{\prime}\right)$.

Выберем произвольное $R_{1}>0$. Каждьй линейный функционал на $\mathbb{R}^{2}$, принимающий целые значения на векторах $v_{1}$ и $v_{2}$, является целочисленной линейной комбинацией функционалов $l_{1}$ и $l_{2}$, заданных соотношениями $l_{1}\left(v_{1}\right)=l_{2}\left(v_{2}\right)=1$, $l_{1}\left(v_{2}\right)=l_{2}\left(v_{1}\right)=0$. Лиш конечное число таких функционалов имеет норму, не превышаюшую $\pi^{-1} R_{1}$. Из леммы 3.1 следует, что произвольный ненулевой линейный функционал на пространстве $\mathbb{R}^{2}$ принимает нецелое значение на некотором векторе $v \in H_{1}$. Тем самым, найдутся векторы $v_{3}, \ldots, v_{n} \in H_{1}$ такие, что каждый ненулевой линейный функционал на $\mathbb{R}^{2}$, норма которого не больше $\pi^{-1} R_{1}$, принимает нецелое значение хотя бы на одном из векторов $v_{1}, \ldots, v_{n}$. Векторы можно выбрать таким образом, чтобы среди векторов $v_{1},-v_{1}, \ldots, v_{n},-v_{n}$ не было двух одинаковых. При этом, как следует из сказанного выше, найдутся натуральное число $M$ и постоянная $I_{2}>0$ такие, что для любого $R>0$ имеет место неравенство $1-\left\|C_{R}^{M}\right\| \geqslant I_{2}\left(1-\sup \left|\widetilde{h}_{R}\right|\right)$, где $\widetilde{h}_{R}=n^{-1} \sum_{j=1}^{n} \cos \left(R\left(\Phi, v_{j}\right)\right)$. Если $\left|\widetilde{h}_{R}(t)\right|=1$ для некоторого $t \in S^{1}$, то числа $\pi^{-1} R\left(\Phi(t), v_{1}\right), \ldots, \pi^{-1} R\left(\Phi(t), v_{n}\right)$ целые. Отображение $v \mapsto \pi^{-1} R(\Phi(t), v)$ является ненулевым линейным функционалом, норма которого равна $\pi^{-1} R$. Тем самьм, при $0<R \leqslant R_{1}$ имеем $\left|\widetilde{h}_{R}\right|<1$ на всей окружности. Поскольку функция $\widetilde{h}_{R}$ непрерывна, $\sup \left|\widetilde{h}_{R}\right|<1$ при $0<R \leqslant R_{1}$. Как следствие, $\left\|C_{R}^{M}\right\|<1$ при $0<R \leqslant R_{1}$. Ввиду произвольности выбора $R_{1}$ семейство операторов $\left\{C_{R}\right\}_{R>0}$ обладает свойством $\left(\mathrm{V} 2^{\prime}\right)$.

Лемма 3.9. Если изометрии $A_{1}, \ldots, A_{k}$ не удовлетворяют условиям (i), (ii) теоремы 1.4, а семейство операторов $C_{R}, R>0$, обладает свойством (V1'), то это же семейство обладает и свойством (V1b). 
ДокАЗАТЕЛЬСтво. Ввиду свойства $\left(\mathrm{V} 1^{\prime}\right)$ найдутся положительные постоянные $I_{1}, R_{0}$ и натуральное число $m$ такие, что $\left\|C_{R}^{m}\right\| \leqslant 1-I_{1} R^{2}$ при $0<R \leqslant R_{0}$.

Декартовы координаты, в которых вычислялись радиальные операторы $C_{R}$, $R>0$, позволяют рассматривать произвольную изометрию из группы $\mathscr{G}$ как преобразование $\mathbb{R}^{2}$. Каждую из изометрий $A_{j}, 1 \leqslant j \leqslant k$, представим в виде $A_{j}=B_{j} T_{j}$, где $B_{j}$ - изометрия, переводящая в себя начало координат, а $T_{j}$ - параллельный перенос на некоторый вектор $v_{j} \in \mathbb{R}^{2}$. Отображение $b_{j}=\Phi^{-1} B_{j} \Phi$ является корректно определенньм преобразованием окружности $S^{1}$ вида $t \mapsto t+\varphi$ или $t \mapsto \varphi-t$, где $\varphi \in S^{1}$. Обозначим через $U_{j}$ унитарный оператор в пространстве $L_{2}\left(S^{1}\right)$, действуюший по правилу $h \mapsto h \circ b_{j}$. Согласно лемме $2.2 u\left[B_{j}\right]_{R}=U_{j}$ для любого $R>0$, а радиальный оператор $u\left[T_{j}\right]_{R}$ является оператором умножения на функцию $e^{i R\left(\Phi, v_{j}\right)}$. Отсюда следует, что $C_{R} h=k^{-1} \sum_{j=1}^{k} e^{i R\left(\Phi, v_{j}\right)} U_{j} h$ для любого $R>0$ и функции $h \in L_{2}\left(S^{1}\right)$. Определим операторы $D_{0}, D_{1}, D_{2}$ в пространстве $L_{2}\left(S^{1}\right)$ посредством формул $D_{0} h=k^{-1} \sum_{j=1}^{k} U_{j} h, D_{1} h=k^{-1} \sum_{j=1}^{k}\left(\Phi, v_{j}\right) U_{j} h$, $D_{2} h=-(2 k)^{-1} \sum_{j=1}^{k}\left(\Phi, v_{j}\right)^{2} U_{j} h, h \in L_{2}\left(S^{1}\right)$. Очевидно, найдется постоянная $I_{2}>0$ такая, что $\left\|C_{R}-\left(D_{0}+i R D_{1}+R^{2} D_{2}\right)\right\| \leqslant I_{2} R^{3}$ при $0<R \leqslant R_{0}$.

Пусть $H_{1}, H_{2}, H_{2}^{+}$- вешественные линейные подпространства пространства $L_{2}\left(S^{1}\right)$, первое из которых порождено функциями $t \mapsto \cos t$ и $t \mapsto \sin t$, второе функциями $t \mapsto \cos 2 t$ и $t \mapsto \sin 2 t$, а третье - функцией 1 и пространством $H_{2}$. Легко видеть, что пространства $H_{1}, H_{2}$ и $H_{2}^{+}$инвариантны относительно оператора $D_{0}$. Произвольная ненулевая функция $f \in H_{1}$ достигает своего максимального значения в единственной точке $t_{0} \in S^{1}$. Для любого целого $j, 1 \leqslant j \leqslant k$, равенство $U_{j} f=f$ имеет место в том и лишш том случае, когда $b_{j}\left(t_{0}\right)=t_{0}$. Равенство $D_{0} f=f$ имеет место в том и лишь том случае, когда $t_{0}$ - общая неподвижная точка отображений $b_{1}, \ldots, b_{k}$. Далее, ненулевая функция $f \in H_{2}$ принимает свое максимальное значение в двух точках $t_{1}, t_{2} \in S^{1}$, расстояние между которыми равно $\pi$. При этом равенство $U_{j} f=f, 1 \leqslant j \leqslant k$, имеет место в том и лишш том случае, когда отображение $b_{j}$ переводит в себя множество $\left\{t_{1}, t_{2}\right\}$. Равенство $D_{0} f=f$ имеет место в том и лишь том случае, когда множество $\left\{t_{1}, t_{2}\right\}$ инвариантно относительно каждого из отображений $b_{1}, \ldots, b_{k}$. Таким образом, если ограничение оператора $1-D_{0}$ на одно из пространств $H_{1}, H_{2}$ вырождено, то каждое из отображений $b_{1}, \ldots, b_{k}$ имеет неподвижную точку или меняет местами две точки, расстояние между которьми равно $\pi$. В любом случае отображения $b_{1}, \ldots, b_{k}$ инволютивны. Тогда операторы $U_{1}, \ldots, U_{k}$ самосопряжены, а с ними самосопряжен и оператор $D_{0}$.

Очевидно, $D_{1} 1=k^{-1}\left(\Phi, v_{1}+\cdots+v_{k}\right) \in H_{1}$. Покажем, что найдется функция $f_{1} \in H_{1}$ такая, что $\left(1-D_{0}\right) f_{1}=D_{1} 1$. Это очевидно, если ограничение оператоpa $1-D_{0}$ на пространство $H_{1}$ невырождено. В противном случае отображения $b_{1}, \ldots, b_{k}$ имеют общую неподвижную точку $t_{0} \in S^{1}$. При этом каждая из изометрий $B_{1}, \ldots, B_{k}$ либо тождественна, либо является осевой симметрией, ось $l$ которой проходит через начало координат и точку $\Phi\left(t_{0}\right)$. Вначале предположим, что все преобразования $B_{1}, \ldots, B_{k}$ тождественны. Если сумма векторов $v_{1}, \ldots, v_{k}$ ненулевая, то изометрии $A_{1}, \ldots, A_{k}$ удовлетворяют условию (i) теоремы 1.4. Если же эта сумма равна нулю, то $D_{1} 1=0$, тогда можно положить $f_{1}=0$. Теперь обратимся к случаю, когда среди изометрий $B_{1}, \ldots, B_{k}$ есть хотя бы одна осевая 
симметрия. Для любой точки $x \in \mathbb{R}^{2}$ ортогональная проекция вектора $A_{j} x-x$, $1 \leqslant j \leqslant k$, на прямую $l$ такая же, как и у вектора $v_{j}$. Тем самым, если сумма векторов $v_{1}, \ldots, v_{k}$ не ортогональна прямой $l$, то изометрии $A_{1}, \ldots, A_{k}$ удовлетворяют условию (ii) теоремы 1.4. Если же эта сумма ортогональна $l$, т.е. $\left(D_{1} 1\right)\left(t_{0}\right)=0$, то $D_{0}\left(D_{1} 1\right)=k^{-1}\left(k-2 k_{1}\right) D_{1} 1$, где $k_{1}$ - число осевых симметрий среди изометрий $B_{1}, \ldots, B_{k}$, и мы можем положить $f_{1}=k\left(2 k_{1}\right)^{-1} D_{1} 1$.

Очевидно, $D_{2} 1 \in H_{2}^{+}$и $D_{1} H_{1} \subset H_{2}^{+}$, поэтому функция $h_{0}=D_{2} 1-D_{1} f_{1}$ принадлежит пространству $H_{2}^{+}$. Обозначим через $\widetilde{D}$ ограничение оператора $1-D_{0}$ на это пространство. Пусть $\widetilde{H}_{0}$ - ядро, а $\widetilde{H}_{2}$ - образ оператора $\widetilde{D}$. Легко видеть, что $1 \in \widetilde{H}_{0}$ и $\widetilde{D} H_{2}=\widetilde{H}_{2}$. Если ограничение оператора $1-D_{0}$ на пространство $H_{2}$ невырождено, то $\widetilde{H}_{2}=H_{2}$, а пространство $\widetilde{H}_{0}$ состоит из постоянных функций. Если же это ограничение вырождено, то, как показано выше, оператор $D_{0}$ самосопряжен, и тогда пространства $\widetilde{H}_{0}$ и $\widetilde{H}_{2}$ ортогональны. В любом случае пространство $H_{2}^{+}$ разлагается в прямую сумму своих подпространств $\widetilde{H}_{0}$ и $\widetilde{H}_{2}$. Как следствие, найдутся функции $g \in \widetilde{H}_{0}$ и $f_{2} \in H_{2}$ такие, что $\left(1-D_{0}\right) f_{2}-g=h_{0}$. При этом $D_{0} g=g$.

Для каждого $R>0$ положим $h_{R}=1+i R f_{1}+R^{2} f_{2}$. Очевидно, найдется постоянная $I_{3}>0$ такая, что $\left\|h_{R}-1\right\| \leqslant I_{3} R$ и $\left\|h_{R}\right\| \leqslant I_{3}$ при $0<R \leqslant R_{0}$. Из соотношений $\left(1-D_{0}\right) 1=0,\left(1-D_{0}\right) f_{1}=D_{1} 1,\left(1-D_{0}\right) f_{2}-g=D_{2} 1-D_{1} f_{1}$ следует, что

$$
\left\|\left(D_{0}+i R D_{1}+R^{2} D_{2}\right) h_{R}-\left(1-g R^{2}\right) h_{R}\right\| \leqslant I_{4} R^{3}
$$

при $0<R \leqslant R_{0}$, где $I_{4}-$ некоторая положительная постоянная. Тогда

$$
\left\|C_{R} h_{R}-\left(1-g R^{2}\right) h_{R}\right\| \leqslant\left(I_{2} I_{3}+I_{4}\right) R^{3}
$$

при $0<R \leqslant R_{0}$.

Произвольные функции $h, \widetilde{h} \in L_{2}\left(S^{1}\right)$ удовлетворяют тождеству

$$
\|h+\widetilde{h}\|^{2}+\|h-\widetilde{h}\|^{2}=2\|h\|^{2}+2\|\widetilde{h}\|^{2} .
$$

Тем самьм, если $\|h\|=\|\widetilde{h}\|$ и $\|h+\widetilde{h}\|=\|h\|+\|\widetilde{h}\|$, то $h=\widetilde{h}$. Это соображение приводит к следующему выводу: для любой функции $h \in L_{2}\left(S^{1}\right)$ равенство норм $\left\|D_{0} h\right\|=\|h\|$ равносильно тому, что функции $U_{1} h, \ldots, U_{k} h$ совпадают. Тогда равенство функций $D_{0} h=h$ равносильно тому, что $U_{j} h=h$ при $j=1, \ldots, k$. Таким образом, функция $g$ инвариантна относительно операторов $U_{1}, \ldots, U_{k}$. Это влечет перестановочность оператора умножения на функцию $g$ с операторами $U_{1}, \ldots, U_{k}$, что, в свою очередь, влечет перестановочность умножения на $g$ с каждым из операторов $C_{R}, R>0$. Кроме того, функция $g$ непрерывна и вещественнозначна, так как $g \in H_{2}^{+}$.

Для завершения доказательства осталось убедиться, что функция $g$ всюду положительна. Очевидно, $\left\|C_{R}\right\| \leqslant 1$ для каждого $R>0$. Поскольку оператор умножения на функцию $g$ коммутирует с оператором $C_{R}$, при $0<R \leqslant R_{0}$ для любого натурального $n$ имеет место оценка

$$
\left\|C_{R}^{n} h_{R}-\left(1-g R^{2}\right) C_{R}^{n-1} h_{R}\right\| \leqslant\left(I_{2} I_{3}+I_{4}\right) R^{3},
$$

откуда следует, что

$$
\left\|C_{R}^{m} h_{R}-\left(1-g R^{2}\right)^{m} h_{R}\right\| \leqslant I_{5} R^{3},
$$


где $I_{5}=m\left(1+R_{0}^{2} \sup |g|\right)^{m-1}\left(I_{2} I_{3}+I_{4}\right)$. Возьмем произвольное $\varepsilon>0$. Положим $E_{\varepsilon}=\left\{t \in S^{1} \mid g(t)<I_{1}-\varepsilon\right\}$. Пусть $\chi_{\varepsilon}-$ функция на $S^{1}$, являющаяся характеристической функцией множества $E_{\varepsilon}$. Для любого $R \in\left(0, R_{0}\right]$ имеет место оценка $\left\|\chi_{\varepsilon} C_{R}^{m} h_{R}-\left(1-g R^{2}\right)^{m} \chi_{\varepsilon} h_{R}\right\| \leqslant I_{5} R^{3}$. Поскольку функция $g$ инвариантна относительно операторов $U_{1}, \ldots, U_{k}$, функция $\chi_{\varepsilon}$ также инвариантна относительно них. Как следствие, оператор $C_{R}$ коммутирует с оператором умножения на $\chi_{\varepsilon}$. В частности, $\chi_{\varepsilon} C_{R}^{m} h_{R}=C_{R}^{m}\left(\chi_{\varepsilon} h_{R}\right)$. Тогда $\left\|\chi_{\varepsilon} C_{R}^{m} h_{R}\right\| \leqslant\left(1-I_{1} R^{2}\right)\left\|\chi_{\varepsilon} h_{R}\right\|$. Кроме того,

$$
\left\|\left(1-g R^{2}\right) \chi_{\varepsilon} h_{R}\right\| \geqslant\left(1-\left(I_{1}-\varepsilon\right) R^{2}\right)\left\|\chi_{\varepsilon} h_{R}\right\|,
$$

так как $1-g R^{2} \geqslant 1-\left(I_{1}-\varepsilon\right) R^{2}$ на множестве $E_{\varepsilon}$. Отсюда следует, что

$$
\varepsilon R^{2}\left\|\chi_{\varepsilon} h_{R}\right\| \leqslant\left\|\left(1-g R^{2}\right) \chi_{\varepsilon} h_{R}\right\|-\left\|\chi_{\varepsilon} C_{R}^{m} h_{R}\right\| \leqslant I_{5} R^{3} .
$$

Тогда $\left\|\chi_{\varepsilon}\right\| \leqslant\left\|\chi_{\varepsilon} h_{R}\right\|+\left\|h_{R}-1\right\| \leqslant\left(\varepsilon^{-1} I_{5}+I_{3}\right) R$ при $0<R \leqslant R_{0}$. Это значит, что $\chi_{\varepsilon}=0$ почти всюду на $S^{1}$. При этом множество $E_{\varepsilon}$ пусто, так как оно открытое. Поскольку $\varepsilon$ можно было выбрать сколь угодно малым, inf $g \geqslant I_{1}>0$.

Для каждого $a \in(0,1)$ определим последовательность $p_{0, a}, p_{1, a}, \ldots$ многочленов рекуррентными соотношениями $p_{0, a}(z)=1, p_{1, a}(z)=z, p_{n+1, a}(z)=(1+a) \times$ $z p_{n, a}(z)-a p_{n-1, a}(z)$ при $n \geqslant 1$.

Лемма 3.10. Многочлены $p_{0, a}, p_{1, a}, \ldots$ удовлетворяют условиям (W1), (W1b) $и$ (W2), сформулированныц в $\S 2$.

ДокАЗАТЕЛЬСтво. Согласно [5; лемма 3.6] семейство многочленов $p_{n, a}, n=$ $1,2, \ldots$, удовлетворяет условиям (W1) и (W2). Добавление к семейству многочлена $p_{0, a}$ эти условия не нарушает. Проверим выполнение условия (W1b). Пусть $z_{a}=2 a^{1 / 2}(1+a)^{-1}$. Для каждого $z \in\left(z_{a}, 1\right]$ и целого $n \geqslant 0$ положим

$$
q_{n}(z)=K_{1}(z) \lambda_{1}^{n}(z)+K_{2}(z) \lambda_{2}^{n}(z)
$$

где

$$
\begin{gathered}
\lambda_{1}(z)=(1+a) \frac{z}{2}+\left((1+a)^{2} \frac{z^{2}}{4}-a\right)^{1 / 2}, \\
\lambda_{2}(z)=(1+a) \frac{z}{2}-\left((1+a)^{2} \frac{z^{2}}{4}-a\right)^{1 / 2}, \\
K_{1}(z)=\frac{z-\lambda_{2}(z)}{\lambda_{1}(z)-\lambda_{2}(z)}, \quad K_{2}(z)=\frac{\lambda_{1}(z)-z}{\lambda_{1}(z)-\lambda_{2}(z)} .
\end{gathered}
$$

При этом $q_{n}(z)=p_{n, a}(z)$. В самом деле, вьполнение условий $q_{0}(z)=1$ и $q_{1}(z)=z$ обеспечено выбором функций $K_{1}, K_{2}$, а рекуррентные соотношения $q_{n+1}(z)=$ $(1+a) z q_{n}(z)-a q_{n-1}(z)$ следуют из того, что $\lambda_{1}(z), \lambda_{2}(z)-$ корни уравнения $\lambda^{2}=(1+a) z \lambda-a$.

Функции $\lambda_{1}, \lambda_{2}, K_{1}, K_{2}$ бесконечно дифференцируемы на промежутке $\left(z_{a}, 1\right]$. Непосредственный подсчет показывает, что $\lambda_{1}(1)=1, \lambda_{1}^{\prime}(1)=(1+a) /(1-a)>0$, 
$\lambda_{2}(1)=a, K_{1}(1)=1, K_{2}(1)=0$. Как следствие, найдутся постоянные $I_{1}>0$ и $\varepsilon \in\left(0,1-z_{a}\right)$ такие, что при $0 \leqslant z \leqslant \varepsilon$ имеют место оценки

$$
\begin{gathered}
0<\lambda_{2}(1-z)<\lambda_{1}(1-z) \leqslant 1, \quad 0<1-\lambda_{1}^{\prime}(1) z \leqslant 1 \\
\left|\lambda_{1}(1-z)-1+\lambda_{1}^{\prime}(1) z\right| \leqslant I_{1} z^{2} \\
\left|K_{1}(1-z)-1\right| \leqslant I_{1} z, \quad\left|K_{2}(1-z)\right| \leqslant I_{1} z .
\end{gathered}
$$

Тогда для любого целого $n \geqslant 0$ на отрезке $[1-\varepsilon, 1]$ имеет место оценка

$$
\left|p_{n, a}-\lambda_{1}^{n}\right| \leqslant\left|K_{1}-1\right|+\left|K_{2}\right|
$$

кроме того, $\left|\lambda_{1}^{n}(1-z)-\left(1-\lambda_{1}^{\prime}(1) z\right)^{n}\right| \leqslant n\left|\lambda_{1}(1-z)-1+\lambda_{1}^{\prime}(1) z\right| \leqslant I_{1} n z^{2}$ при $0 \leqslant z \leqslant \varepsilon$. Тем самьм, $\left|p_{n, a}(1-z)-\left(1-\lambda_{1}^{\prime}(1) z\right)^{n}\right| \leqslant 2 I_{1} z+I_{1} n z^{2}$ при $0 \leqslant z \leqslant \varepsilon$. Условие (W1b) проверено.

ДОКАЗАТЕЛЬСТВО ТЕОРЕМЫ 1.4. Пусть $A_{1}, \ldots, A_{k}$ - изометрии плоскости $P$, которые не имеют общей неподвижной точки и не переводят в себя одну и ту же решетку вида $\mathscr{L}_{Q}$, где $Q$ - полоса, квадрат или правильный треугольник. Обозначим через $\mathscr{H}$ полугруппу, порожденную изометриями $A_{1}, \ldots, A_{k}$. Положим $C=k^{-1}\left(u\left[A_{1}\right]+\cdots+u\left[A_{k}\right]\right)$. Введем на плоскости $P$ декартовы координаты $\xi$. Пусть $\left\{C_{R}\right\}_{R>0}$ - семейство радиальных операторов, соответствующих оператору $C$ в системе координат $\xi$. Если изометрии $A_{1}, \ldots, A_{k}$ удовлетворяют одному из условий (i), (ii) теоремы 1.4 , то ввиду леммы 3.6 действие $\mathrm{FSG}_{k}\left[A_{1}, \ldots, A_{k}\right]$ удовлетворяет условию 2 ) той же теоремы. В противном случае из лемм 3.4 и 3.5 следует, что полугруппа $\mathscr{H}$ содержит поворот на угол, несоизмеримый с $\pi$, или является группой. Тогда из лемм 3.7 и 3.8 следует, что семейство операторов $\left\{C_{R}\right\}_{R>0}$ обладает свойствами $\left(\mathrm{V} 1^{\prime}\right)$ и $\left(\mathrm{V} 2^{\prime}\right)$. Наличие у этого семейства свойства $(\mathrm{V} 1 \mathrm{~b})$ следует из леммы 3.9. Далее, из леммы 2.2 следует, что оператор $C_{R}$ непрерывно зависит от параметра $R$. Кроме того, $\left\|C_{R}\right\| \leqslant 1$, так как $C_{R}$ - среднее арифметическое нескольких унитарных операторов. Тем самьм, семейство операторов $\left\{C_{R}\right\}_{R>0}$ удовлетворяет всем условиям предложения 2.5. Согласно этому предложению семейство операторов $C_{R}^{n}, R>0, n=0,1, \ldots$, обладает свойствами $(\mathrm{P} 1),(\mathrm{P} 1 \mathrm{~b})$ и $(\mathrm{P} 2)$. Заметим, что операторы $C_{R}^{n}, R>0$, суть радиальные операторы, соответствующие оператору $C^{n}$ в системе координат $\xi$. В свою очеред, операторы $1, C, C^{2}, \ldots$ суть усредняющие операторы, сопоставляемые действию $\mathrm{FSG}_{k}\left[A_{1}, \ldots, A_{k}\right]$. Ввиду предложения 2.3 это действие удовлетворяет условию 1$)$ теоремы 1.4. Этим завершено доказательство теоремы 1.4.

ДОКАЗАТЕЛЬСТвО ТЕОРЕМЫ 1.3. Каковы бЫ Ни бЫли изометрии $A_{1}, \ldots, A_{k}$, преобразования $A_{1}, A_{1}^{-1}, \ldots, A_{k}, A_{k}^{-1}$ не удовлетворяют условиям (i), (ii) теоремы 1.4. Тем самым, утверждение теоремы 1.3 в части, касаюшейся действий вида $\mathrm{FSG}_{2 k}\left[A_{1}, A_{1}^{-1}, \ldots, A_{k}, A_{k}^{-1}\right]$, является следствием теоремы 1.4. Рассмотрим действие $d=G\left[A_{1}, \ldots, A_{k}\right]$, где $G=\mathrm{FG}_{k}$ или (при условии, что $A_{1}, \ldots, A_{k}-$ инволюции) $G=\mathbb{Z}_{2}^{* k}$. Положим $a=(2 k-1)^{-1}$, если действует группа $\mathrm{FG}_{k}$, и $a=(k-1)^{-1}$, если действует $\mathbb{Z}_{2}^{* k}$. Заметим, что $k \geqslant 2$, а если $A_{1}, \ldots, A_{k}$ - инволюции, то $k \geqslant 3$, так как иначе изометрии $A_{1}, \ldots, A_{k}$ имеют общую неподвижную 
точку или переводят в себя некоторую решетку, порожденную полосой. В частности, $0<a<1$. Усредняющие операторы, сопоставляемые действию $d$, имеют вид $C^{(n)}=p_{n, a}(D)$, где $D=(2 k)^{-1}\left(u\left[A_{1}\right]+u\left[A_{1}^{-1}\right]+\cdots+u\left[A_{k}\right]+u\left[A_{k}^{-1}\right]\right)($ если $A_{1}, \ldots, A_{k}$ - инволюции, то $\left.D=C=k^{-1}\left(u\left[A_{1}\right]+\cdots+u\left[A_{k}\right]\right)\right)$. В самом деле, равенства $C^{(0)}=1$ и $C^{(1)}=D$ очевидны, рекуррентные же соотношения $C^{(n+1)}=(1+a) D C^{(n)}-a C^{(n-1)}, n=1,2, \ldots$, проверяются без особых затруднений (ср. доказательство теорем 1.1 и 1.2 из [5]). Ввиду леммы $2.2 C_{R}^{(n)}=$ $p_{n, a}\left(D_{R}\right)$ для любого $R>0$ и целого $n \geqslant 0$. Изометрии $A_{1}, A_{1}^{-1}, \ldots, A_{k}, A_{k}^{-1}$ не удовлетворяют ни одному из условий (i), (ii) теоремы 1.4 , а порожденная ими полугруппа изометрий, естественно, является группой. Тогда из лемм 3.8 и 3.9 следует, что семейство операторов $\left\{D_{R}\right\}_{R>0}$ обладает свойствами $\left(\mathrm{V} 1^{\prime}\right),(\mathrm{V} 1 \mathrm{~b})$ и $\left(\mathrm{V}^{\prime}\right)$. Оператор $D_{R}$ непрерьвно зависит от параметра $R$ и самосопряжен, так как $D_{R}=\left(C_{R}+C_{R}^{*}\right) / 2$. При этом $\left\|D_{R}^{n}\right\|=\left\|D_{R}\right\|^{n}$ для любого натурального $n$. Отсюда следует, что для семейства $\left\{D_{R}\right\}_{R>0}$ свойство $\left(\mathrm{V} 1^{\prime}\right)$ эквивалентно свойству (V1), а свойство $\left(\mathrm{V} 2^{\prime}\right)$ - свойству (V2). Таким образом, это семейство удовлетворяет всем условиям предложения 2.4. Многочлены $p_{0, a}, p_{1, a}, \ldots$ имеют вещественные коэффициенты и удовлетворяют условиям (W1), (W1b), (W2) ввиду леммы 3.10. Согласно предложению 2.4 семейство радиальных операторов $C_{R}^{(n)}$, $R>0, n=0,1, \ldots$, обладает свойствами $(\mathrm{P} 1),(\mathrm{P} 1 \mathrm{~b})$ и $(\mathrm{P} 2)$. Применив предложение 2.3, мы завершаем доказательство теоремы 1.3 .

\section{Список литературы}

1. Арнольд В.И., Крылов А. Л. Равномерное распределение точек на сфере и некоторые эргодические свойства решений линейных обыкновенных дифференциальных уравнений в комплексной области // Докл. АН СССР. 1963. Т. 148. № 1. С. 9-12.

2. Каждан Д. А. Равномерное распределение на плоскости // Труды ММО. 1965. Т. 14. C. 299-305.

3. Guivarc' $h$ Y. Équirepartition dans les espaces homogenes // Lecture Notes in Math. 1976. V. 532. P. 131-142.

4. Воробец Я.Б. О равномерном распределении орбит действий свободных групп и полугрупп на плоскости // Труды МИАН. 2000. Т. 231. С. 64-95.

5. Воробеи Я. Б. О действиях конечно порожденных групп и полугрупп на плоскости посредством изометрий // Матем. заметки. 2004. (в печати).

Институт прикладных проблем механики

Поступила в редакцию и математики им. Я. С. Пидстрьгача 18.04.2003

НАН Украины, Львов

E-mail : vorobets@complex.lviv.ua 